\title{
A High-Fidelity Method to Analyze Perturbation Evolution in Turbulent Flows
}

\author{
S. Unnikrishnan ${ }^{\mathrm{a}}$, Datta V. Gaitonde ${ }^{\mathrm{a}, *}$ \\ ${ }^{a}$ Mechanical and Aerospace Engineering, The Ohio State University, Columbus, OH 43210
}

\begin{abstract}
Small perturbation propagation in fluid flows is usually examined by linearizing the governing equations about a steady basic state. It is often useful, however, to study perturbation evolution in the unsteady evolving turbulent environment. Such analyses can elucidate the role of perturbations in the generation of coherent structures or the production of noise from jet turbulence. The appropriate equations are still the linearized Navier-Stokes equations, except that the linearization must be performed about the instantaneous evolving turbulent state, which forms the coefficients of the linearized equations. This is a far more difficult problem since in addition to the turbulent state, its rate of change and the perturbation field are all required at each instant. In this paper, we develop and use a novel technique for this problem by using a pair (denoted "baseline" and "twin") of simultaneous synchronized Large-Eddy Simulations $(L E S)$. At each time-step, small disturbances whose propagation characteristics are to be studied, are introduced into the twin through a forcing term. At subsequent time steps, the difference between the two simulations is shown to be equivalent to solving the forced Navier-Stokes equations, linearized about the instantaneous turbulent state. The technique does not put constraints on the forcing, which could be arbitrary, e.g., white noise or other stochastic variants. We consider, however, "native" forcing having properties of disturbances that exist naturally in the turbulent environment. The method then isolates the effect of turbulence in a particular region on the rest of the field, which is useful in the study of noise source localization. The synchronized technique is relatively simple to implement into existing codes. In addition to minimizing the storage and retrieval of large time-varying datasets, it avoids the need to explicitly linearize the governing equations, which can be a very complicated task for viscous terms or turbulence closures. The method is illustrated by application to a well-validated Mach 1.3 jet. Specifically, the effects of turbulence on the jet lipline and core collapse regions on the near-acoustic fields are isolated. The properties of the method, including linearity and effect of initial transients, are discussed. The results provide insight into how turbulence from different parts of the jet contribute to the observed dominance of low and high frequency content at shallow and sideline angles, respectively.
\end{abstract}

\footnotetext{
${ }^{*}$ Corresponding author at: Mechanical and Aerospace Engineering, The Ohio State University, Columbus, $\mathrm{OH} 43210$

Email addresses: sasidharannair.1@osu.edu (S. Unnikrishnan), gaitonde.3@osu.edu (Datta V. Gaitonde)
}

Preprint submitted to Journal of Computational Physics

November 30, 2015

(C) 2016. This manuscript version is made available under the Elsevier user license http://www.elsevier.com/open-access/userlicense/1.0/ 
Keywords: Large-Eddy Simulations, Linearized Navier-Stokes, Perturbation evolution, Acoustic Analysis

\section{Introduction}

The evolution of small perturbations has significant implications on the dynamics of laminar and turbulent flow-fields. Such studies can provide insight into stability properties, and generate insight into control techniques. The basic state is usually steady.

5 The governing equations are either the Euler or Navier-Stokes equations for inviscid or viscous flows respectively. Linearization starts by splitting the flow variables into mean and perturbation quantities, and assuming that the products of the latter are small. The basic state is further assumed to satisfy the governing equations. Judicious assumptions on the basic state, such as parallel or slowly varying streamwise change, greatly 10 simplify the task of solving the equations. Popular approaches use modal solutions, and include linear stability theory [1, 2, 3] and parabolized stability theory [4]. Global stability theory [5, 6, 7] removes many of these assumptions. Comprehensive reviews of such techniques may be found in Theofilis [8].

In recent years, such techniques have also been applied to turbulent mean flows, even though these do not satisfy the governing equations. For example, many of the features associated with the low frequency modes observed in shock/turbulent boundary layer interactions have been successfully reproduced by perturbing the time-averaged flow obtained from a Large-Eddy Simulation (LES) [9]. In the area of jet noise, both experimental and simulated mean turbulent fields have been subjected to modal analysis, and many of the important features of the acoustic field have been elucidated through a wave packet framework [10, 11, 12].

Although very insightful, mean flow analyses do not provide detailed understanding of how the underlying turbulent flow processes disturbances, since the mean flow washes out coherent structures $(C S)$ and time-accurate phase information is lost. The

25 CS-turbulence interaction is however a crucial aspect of the dynamics [13]. Hussain and Stout [14] specifically call out aircraft trailing vortices and wind turbine blades, but clearly these dynamics are important to all turbulent phenomena.

Another area where key insights can be obtained by examining disturbance processing by turbulent flows is acoustics, such as in the generation of jet noise. The connection between turbulent fluctuations and noise was established by Lighthill [15, 16] and various studies have blended experiments and computation to provide significant insight into the overall phenomenology [17, 18, 19, 20, 21]. These efforts have successfully obtained key conclusions on the different effects of small versus large structures on the noise field, and have also identified regions in the jet that best correlate with the 35 farfield.

Nonetheless, considerable uncertainty exists about the precise mechanics by which the large amount of energy in the jet is filtered to the relatively small, yet highly destructive, energy in the primarily acoustic field outside the turbulent region. One way to make progress is to use simulations to notionally tag the turbulent fluctuation in a 40 region of interest, and to then track the "slight phase imperfections from the compressibility of the flow" [22] that yield acoustic pressure fluctuations. Similar to methods 
that examine perturbation propagation through steady basic states, such an analysis may be performed for a time-varying basic state by linearizing the Navier-Stokes equations about the unsteady instantaneous turbulent state. Our overall goal in this paper is to propose and demonstrate such a capability.

Numerous studies have examined disturbance propagation using steady basic states. For example, Mankbadi et al. [23] show that the linearized Euler equations (LEE) can reproduce elements of jet noise when forced with random signals. Other interesting results with LEE include those of Bailly and Juve [24], who develop a stochastic noise

50 generation and radiation model and Prax et al. [25] who discuss the use of sources to control the growth of the hydrodynamic mode.

There are relatively fewer examples of the use of Steady basic state linearized Navier-Stokes equations, which we abbreviate as $S$-LNS. Kierkegaard [26] solves the $S$-LNS equations in the frequency domain to obtain an industrial tool for duct aeroa55 coustics, while Jovanović and Bamieh [27] explore impulse forcing in duct flows to delineate "rich and complex structures hitherto unseen by other analysis methods." $S$ $L N S$ have also been employed with time-varying forcing to mimic some of the effects of an unsteady basic state. Insights from the response of $S-L N S$ have been leveraged for suppression of turbulence [28]. Another example of the use of $S$-LNS is to repro-

60 duce the statistics of a Direct Numerical Simulation $(D N S)$ with a simpler procedure [29], which is useful for optimal control analysis. Reynolds-averaged Navier-Stokes $(R A N S)$ solutions have also been perturbed to yield global stability results for airfoil buffet onset in good agreement with experiments [30].

Early analyses of unsteady basic states were theoretical in nature (see e.g., Davis

65 [31]). However numerical methods are seeing increased attention, albeit with significant restrictions on the nature of the basic state or the forcing. For example, Burde et al. [32] develop techniques using separation of variables to study the linearized equations in simple unsteady incompressible flows. More recently, Towne et al. [33] use LNS with "jittering", to more accurately predict the acoustic radiation of jets with the wave to packet formulation.

In the present work, we seek to develop a method capable of simulating small disturbance propagation through the unsteady evolving turbulent environment. The phenomena are governed by the Navier-Stokes equations linearized about the evolving turbulent solution. We term these equations Turbulent basic state LNS (T- LNS). 75 Considerations in the derivation of T-LNS are presented in Section 2 the primary difference from $S$-LNS lies in the time derivative. There are however significant practical difficulties in solving T-LNS compared to $S$-LNS. To advance the solution at each time instant, in addition to the state of the disturbance itself, the turbulent solution as well as its rate of change must be known. Computing these a priori and using the stored values quickly leads to severe data management difficulties for the fully $3-D$ problems of interest. Furthermore, while the linearization is straightforward for the inviscid terms, it is less so for the viscous terms if the viscosity is a strong function of temperature, or if a complicated or implicit turbulence closure is used.

The proposed method leverages existing LES codes to overcome these difficulties. 85 It is based on using two simultaneous synchronized LES, which we denote as "baseline" and "twin" (Section 2.1). The latter differs from the former because of the forcing and its consequences. The difference at any instant between the two simulations is shown to 




Figure 1: Description of Synchronized LES algorithm. The vertical dotted arrow on the left indicates progress of time.

be the solution of the forced $T$ - LNS. The forcing function is chosen to fulfill the desired objective, and is typically applied in a localized region. Stochastic and deterministic . processes different frequencies. An alternative of more direct interest in this work is to examine the effect of turbulence in some desired region on the surrounding flowfield. In this case, an appropriate forcing simply boosts, by a very small amount, the existing ("native") turbulent fluctuation in the region of interest in the twin (Section 2.2).

Following the description of the method, we apply it to analyze disturbance propagation in a turbulent Mach 1.3 jet. In Section 3.1 we describe the LES method used, the flow and grid parameters and summarize the validation data. In Section 3.2, we identify select regions along the lipline and the centerline as sample locations to introduce native forcing. The properties of the perturbation field for the different forcing regions and their impact on the near-acoustic field are discussed in Section 3.3 followed by analysis of the main properties of the method, including linearity, effect of initial transients and boundaries of the forcing regions (Section 3.4). Finally, in Section 3.5, the statistical properties of the perturbation field are presented with the goal of clarifying some of the mechanisms by which the experimentally and computationally observed near-acoustic field is established.

\section{Methodology of Synchronized $L E S$}

\subsection{Basic procedure}

As noted earlier, the procedure employs two simulations whose difference yields the desired T-LNS solution. The steps are shown schematically in Figure 1. The left vertical arrow denotes the time axis. The baseline turbulent simulation is first advanced 
in time by itself, until it reaches a statistically stationary asymptotic state ( $\left.T=t_{s}\right)$. Any suitable method may be employed: the specific LES approach used in this work is discussed in Section 3.1. Mean statistics are then acquired until $T=t_{\text {SLES }}$. Since the basic state is itself turbulent, henceforth we distinguish between "fluctuation" and "perturbation". The former is associated with unsteadiness due to turbulence, and is the difference between the instantaneous turbulent flow quantity and its mean. The latter is the signal at any point in the twin due to the forcing applied to the turbulent flow, as further clarified below. The synchronized LES procedure comprises the following steps:

1. A second simulation, denoted "twin" is initialized, using the same state as the baseline at $t_{\text {SLES }}$ and the region of forcing (or window) is identified.

2. At each subsequent time step, the desired forcing, which can be arbitrarily specified, is applied to the twin. In the particular case of native forcing (see below), this requires communication between the baseline and the twin as shown schematically in Figure 1

3. Both the baseline and twin simulations are advanced in time simultaneously using the same time-step size, with the forcing being introduced at each time-step into the twin simulation.

4. The difference of the baseline and twin flowfields at any instant represents the solution of the T-LNS under the effect of the forcing. This difference field will be denoted the "perturbation" field in the discussion below

The terminology thus uses "fluctuation" to characterize the unsteadiness of the turbulent baseline flow, "forcing" to inject the desired disturbances in a region of the twin domain and "perturbation", which represents the effect of forcing on the turbulent flow.

\subsection{Forcing considerations}

The forcing can be arbitrary, similar to the stochastic and time varying techniques used for example in Mankbadi et al. [23] and Jovanovic and Bamieh [29]. In this case, the scaled forcing can be determined and simply added to the flow variables in the twin within a specified region. Our current focus is on exploring the instantaneous interaction of turbulence in a region of interest with its surroundings. This can be accomplished with native forcing. We introduce the Reynolds decomposition to the baseline flow:

$$
\Phi^{b}=\overline{\boldsymbol{\Phi}}^{\mathrm{b}}+\boldsymbol{\phi}^{\prime} \mathbf{b}
$$

where $\Phi$ is the solution vector, superscript b refers to the baseline (unsteady) LES state, $\overline{\boldsymbol{\Phi}}^{\mathbf{b}}$ is the (steady) mean and $\boldsymbol{\phi}^{\prime} \mathbf{b}$ is the corresponding fluctuation. The average, $\bar{\Phi}^{\mathrm{b}}$ is obtained over 150 characteristic time-intervals after the baseline simulation has reached a statistically stationary state. This interval is indicated in Figure 1 $\left(t_{s} \leqslant t \leqslant t_{S L E S}\right)$. Hence, the mean is a precomputed, known value which is invariant over the entire synchronized simulation time. The fluctuations obtained by subtracting the instantaneous from the mean thus include all naturally present scales in the flowfield, including both fine-scale, relatively higher frequency turbulence and any relatively lower frequency coherent features. This aspect is further 
explained in Section 3.2. Native perturbation-boost or forcing $\left(\Delta \Phi^{p}\right)$ is then defined as:

$$
\Delta \boldsymbol{\Phi}^{\mathbf{p}}=f\left(\boldsymbol{\Phi}^{\mathbf{b}}-\overline{\boldsymbol{\Phi}}^{\mathbf{b}}\right)=\epsilon \times \mathscr{W}(\mathbf{X}, t) \times\left(\boldsymbol{\Phi}^{\mathbf{b}}-\overline{\boldsymbol{\Phi}}^{\mathbf{b}}\right)
$$

The "native" nature of $\Delta \Phi^{p}$ is thus evident, since it is generated from the fluctuation, $\left(\boldsymbol{\Phi}^{\mathbf{b}}-\overline{\boldsymbol{\Phi}}^{\mathbf{b}}\right) . \mathscr{W}(\mathbf{X}, t)$ is the spatial window that localizes the forcing to the region whose influence is desired. For the present work, the forcing regions are essentially points in the domain as discussed in Section 3.2. The effect of a diffuse source is also 140 analyzed in Section 3.4 The spatial window representation in Eq. 2 is a general form that can localize both space and time as desired. The space dependence allows focus on specific regions such as point regions or loci of interest, while the time dependence enables finite time forcing. In the current study, only a small subset of possibilities is considered, where the forcing is focused on selected points in the domain and continued for all time. $\epsilon$ is a scaling factor, and should be small enough for linearity, which also ensures that the statistical properties of the baseline and twin simulations remain the same. Numerical tests with native perturbations on the jet flow of Section 3 indicate that $\epsilon$ values ranging from $10^{-3}$ to $10^{-9}$ are appropriate: a value of $10^{-6}$ has been used in the simulations below.

Since native forcing is based on the fluctuation, it does not inject net mass, momentum or energy into the twin. Likewise, the forcing exhibits the same acoustic, entropic and hydrodynamic modes already existing in the environment and does not introduce foreign spectral components into the flow. To maintain the native character of the forcing, the scaling factor $\epsilon$ must be the same for all variables forced in the twin. While different values of $\epsilon$ could be used to examine the effect of, say, boosting the pressure or vortical component only, that option has not been explored here.

In the simulations performed to date, the forcing is applied at every time instant. An impulse response, such as the one discussed by Sinha et al. [11] in the context of Parabolized Stability Equation analysis of the mean flow, could be obtained by applying the forcing only at one or a few time-steps. However, since the basic state is turbulent, the response would then have to be examined in an ensemble sense i.e., repeating the impulse at different realizations of the baseline. This option is also outside the scope of the present work.

During the forcing of the twin simulation, there are two possible sources of numerical contamination. The first is the discontinuity in time caused due to the transients set up while starting the forcing in the twin. This effect is studied in Section 3.4. The second source of numerical contamination is the truncation of the spatial domain of forcing. The associated effects are discussed in Section 3.5 . The results indicate that these errors are small and the inferences are correct.

\subsection{Connection to $T$ - $L N S$}

We now show that the above approach, i.e., the difference between the twin and the baseline is in fact the perturbation as obtained from a solution of the T-LNS. First a detailed analysis is performed using just the inviscid components of the Navier-Stokes equations, represented by the Euler equations. The effects of viscous and turbulence 
terms are summarized later. The Euler equations are:

$$
\begin{gathered}
\frac{\partial(\rho)}{\partial t}+\frac{\partial(\rho u)}{\partial x}+\frac{\partial(\rho v)}{\partial y}+\frac{\partial(\rho w)}{\partial z}=F_{\rho} \\
\frac{\partial(\rho u)}{\partial t}+\frac{\partial\left(\rho u^{2}\right)}{\partial x}+\frac{\partial(\rho u v)}{\partial y}+\frac{\partial(\rho u w)}{\partial z}=-\frac{\partial(p)}{\partial x}+F_{x} \\
\frac{\partial(\rho v)}{\partial t}+\frac{\partial(\rho u v)}{\partial x}+\frac{\partial\left(\rho v^{2}\right)}{\partial y}+\frac{\partial(\rho v w)}{\partial z}=-\frac{\partial(p)}{\partial y}+F_{y} \\
\frac{\partial(\rho w)}{\partial t}+\frac{\partial(\rho u w)}{\partial x}+\frac{\partial(\rho v w)}{\partial y}+\frac{\partial\left(\rho w^{2}\right)}{\partial z}=-\frac{\partial(p)}{\partial z}+F_{z} \\
\frac{\partial(\rho e)}{\partial t}+\frac{\partial[(\rho e+p) u]}{\partial x}+\frac{\partial[(\rho e+p) v]}{\partial y}+\frac{\partial[(\rho e+p) w]}{\partial z}=F_{e}
\end{gathered}
$$

where $u, v, w$ are the Cartesian velocity components, $p$ is the pressure, $\rho$ is the density and $e$ is the total energy. A function $\mathbf{F}$, has been introduced to represent forcing in the twin: $\mathbf{F}=\left\{F_{\rho}, F_{x}, F_{y}, F_{z}, F_{e}\right\}$. In the baseline, $\mathbf{F}=0$.

Unlike $S$ - $L N S$, where linearization is initiated by splitting the flow variables into mean and perturbation values, here we replace the former by the unsteady LES state. Specifically, we consider

$$
\begin{aligned}
& \mathrm{u}(x, y, z, t)=\mathrm{u}_{\mathrm{L}}(x, y, z, t)+\mathrm{u}^{\prime}(x, y, z, t) \\
& v(x, y, z, t)=V_{\mathrm{L}}(x, y, z, t)+v^{\prime}(x, y, z, t) \\
& w(x, y, z, t)=W_{\mathrm{L}}(x, y, z, t)+w^{\prime}(x, y, z, t) \\
& p(x, y, z, t)=P_{L}(x, y, z, t)+p^{\prime}(x, y, z, t) \\
& \rho(x, y, z, t)=\rho_{\mathrm{L}}(x, y, z, t)+\rho^{\prime}(x, y, z, t)
\end{aligned}
$$

where quantities with the subscript (L) represent the unsteady instantaneous LES flow variables and quantities with the superscript $\left({ }^{\prime}\right)$ are the perturbations (not fluctuations).

Substituting into the governing equations, and focusing for brevity on the x-momentum equation, the twin simulation solves:

$$
\frac{\partial(\rho u)}{\partial t}+\frac{\partial\left(\rho u^{2}\right)}{\partial x}+\frac{\partial(\rho u v)}{\partial y}+\frac{\partial(\rho u w)}{\partial z}=-\frac{\partial(p)}{\partial x}+F_{x}
$$

where $F_{x}$ is the $x$-momentum component of the forcing. Upon substituting the decomposition in Eq. 8 into the above $x$-momentum equation, neglecting the nonlinear perturbation terms and subtracting the baseline equation, we obtain the 
equation representing the synchronized $L E S$ procedure as:

$$
\begin{aligned}
& \frac{\partial\left[\rho_{\mathrm{L}} u^{\prime}+\rho^{\prime} \mathrm{U}_{\mathrm{L}}\right]}{\partial t}+\frac{\partial\left[2 \rho_{\mathrm{L}} \mathrm{U}_{\mathrm{L}} u^{\prime}+\rho^{\prime} \mathrm{U}_{\mathrm{L}}^{2}+p^{\prime}\right]}{\partial x} \\
& \quad+\frac{\partial\left[\rho^{\prime} \mathrm{U}_{\mathrm{L}} \mathrm{V}_{\mathrm{L}}+\rho_{\mathrm{L}} \mathrm{U}_{\mathrm{L}} v^{\prime}+\rho_{\mathrm{L}} \mathrm{V}_{\mathrm{L}} u^{\prime}\right]}{\partial y}+\frac{\partial\left[\rho^{\prime} \mathrm{U}_{\mathrm{L}} W_{\mathrm{L}}+\rho_{\mathrm{L}} \mathrm{U}_{\mathrm{L}} w^{\prime}+\rho_{\mathrm{L}} W_{\mathrm{L}} u^{\prime}\right]}{\partial z}=\mathrm{F}_{x}
\end{aligned}
$$

Equation 9 represents the linearized inviscid $\mathrm{x}$-momentum equation for the perturbations subjected to coefficients which are the known unsteady baseline $L E S$ quantities. Upon following a similar procedure for the continuity, energy and the other momentum equations, the linearized system of unsteady inviscid equations are obtained:

$$
\begin{aligned}
& \frac{\partial}{\partial t}\left[\begin{array}{c}
\rho^{\prime} \\
\mathrm{U}_{\mathrm{L}} \rho^{\prime}+\rho_{\mathrm{L}} u^{\prime} \\
\mathrm{V}_{\mathrm{L}} \rho^{\prime}+\rho_{\mathrm{L}} v^{\prime} \\
W_{\mathrm{L}} \rho^{\prime}+\rho_{\mathrm{L}} w^{\prime} \\
e_{\mathrm{L}} \rho^{\prime}+\rho_{\mathrm{L}} e^{\prime}
\end{array}\right]+\frac{\partial}{\partial x}\left[\begin{array}{c}
\mathrm{U}_{\mathrm{L}} \rho^{\prime}+\rho_{\mathrm{L}} u^{\prime} \\
\mathrm{u}_{\mathrm{L}}^{2} \rho^{\prime}+2 \rho_{\mathrm{L}} \mathrm{U}_{\mathrm{L}} u^{\prime}+p^{\prime} \\
\mathrm{u}_{\mathrm{L}} \mathrm{V}_{\mathrm{L}} \rho^{\prime}+\rho_{\mathrm{L}} V_{\mathrm{L}} u^{\prime}+\rho_{\mathrm{L}} \mathrm{U}_{\mathrm{L}} v^{\prime} \\
\mathrm{u}_{\mathrm{L}} W_{\mathrm{L}} \rho^{\prime}+\rho_{\mathrm{L}} W_{\mathrm{L}} u^{\prime}+\rho_{\mathrm{L}} \mathrm{U}_{\mathrm{L}} w^{\prime} \\
\mathrm{u}_{\mathrm{L}} e_{\mathrm{L}} \rho^{\prime}+\left(\rho_{\mathrm{L}} e_{\mathrm{L}}+p_{\mathrm{L}}\right) u^{\prime}+\rho_{\mathrm{L}} \mathrm{U}_{\mathrm{L}} e^{\prime}+\mathrm{U}_{\mathrm{L}} p^{\prime}
\end{array}\right] \\
& +\frac{\partial}{\partial y}\left[\begin{array}{c}
V_{\mathrm{L}} \rho^{\prime}+\rho_{\mathrm{L}} v^{\prime} \\
\mathrm{u}_{\mathrm{L}} \mathrm{V}_{\mathrm{L}} \rho^{\prime}+\rho_{\mathrm{L}} \mathrm{V}_{\mathrm{L}} \mathrm{u}^{\prime}+\rho_{\mathrm{L}} \mathrm{U}_{\mathrm{L}} v^{\prime} \\
\mathrm{V}_{\mathrm{L}}^{2} \rho^{\prime}+2 \rho_{\mathrm{L}} \mathrm{V}_{\mathrm{L}} v^{\prime}+\mathrm{p}^{\prime} \\
\mathrm{V}_{\mathrm{L}} \mathrm{W}_{\mathrm{L}} \rho^{\prime}+\rho_{\mathrm{L}} W_{\mathrm{L}} v^{\prime}+\rho_{\mathrm{L}} \mathrm{V}_{\mathrm{L}} w^{\prime} \\
\mathrm{V}_{\mathrm{L}} e_{\mathrm{L}} \rho^{\prime}+\left(\rho_{\mathrm{L}} e_{\mathrm{L}}+p_{\mathrm{L}}\right) v^{\prime}+\rho_{\mathrm{L}} \mathrm{V}_{\mathrm{L}} e^{\prime}+\mathrm{V}_{\mathrm{L}} p^{\prime}
\end{array}\right] \\
& +\frac{\partial}{\partial z}\left[\begin{array}{c}
W_{\mathrm{L}} \rho^{\prime}+\rho_{\mathrm{L}} w^{\prime} \\
\mathrm{U}_{\mathrm{L}} W_{\mathrm{L}} \rho^{\prime}+\rho_{\mathrm{L}} W_{\mathrm{L}} u^{\prime}+\rho_{\mathrm{L}} \mathrm{U}_{\mathrm{L}} w^{\prime} \\
V_{\mathrm{L}} W_{\mathrm{L}} \rho^{\prime}+\rho_{\mathrm{L}} W_{\mathrm{L}} v^{\prime}+\rho_{\mathrm{L}} V_{\mathrm{L}} w^{\prime} \\
W_{\mathrm{L}}^{2} \rho^{\prime}+2 \rho_{\mathrm{L}} W_{\mathrm{L}} w^{\prime}+p^{\prime} \\
W_{\mathrm{L}} e_{\mathrm{L}} \rho^{\prime}+\left(\rho_{\mathrm{L}} e_{\mathrm{L}}+p_{\mathrm{L}}\right) w^{\prime}+\rho_{\mathrm{L}} W_{\mathrm{L}} e^{\prime}+W_{\mathrm{L}} p^{\prime}
\end{array}\right]=\mathbf{F}
\end{aligned}
$$

Thus, the time-derivative and inviscid components of the Navier-Stokes equations are implicitly linearized by the procedure. Note that the form of the spatial derivative terms is similar to what is obtained when linearization is carried out about a mean basic state, except that the coefficients are now associated with the time-varying turbulent state. However, the time-derivative term is more complicated, and includes rates of change of the evolving turbulent flow. Also it is not necessary to know the explicit form of $\mathbf{F}$ here: its effect on the variables in the forcing region of the twin can be directly prescribed. The forcing component need not be a solution of the Navier-Stokes equations, as any signal of interest can be used to model $\mathrm{F}$ explicitly for non-native forcing. The twin propagates the spectrum of the forcing signal based on the dynamics of the surrounding turbulent flow, yielding the properties of the perturbation field. The native forcing is one choice made to provide a way to track natural perturbations.

The synchronized LES method becomes even more attractive when viscous and turbulence modeling terms are considered. Even if temperature dependence of viscosity $(\mu)$ is neglected, so that the viscous terms in the momentum equation and the thermal 
diffusion terms in the energy equation become linear (constant Prandtl number), the dissipation terms still require special care when explicit linearization is performed. For example, a typical viscous component in the $x$-derivative of the conservative form of the energy equation is:

$$
\frac{\partial}{\partial x}\left[\tau_{x x} u+\tau_{x y} v+\tau_{x z} w\right]
$$

Following the same procedure as for the Euler equations i.e., substituting the decomposition into an $L E S$ basic state and a perturbation, neglecting products of disturbances and subtracting the baseline terms yields:

$$
\begin{array}{r}
\frac{2}{3} \mu \frac{\partial}{\partial x}\left[2 \mathrm{U}_{\mathrm{L}} \frac{\partial u^{\prime}}{\partial x}+2 u^{\prime} \frac{\partial \mathrm{U}_{\mathrm{L}}}{\partial x}-\mathrm{U}_{\mathrm{L}} \frac{\partial v^{\prime}}{\partial y}-u^{\prime} \frac{\partial \mathrm{V}_{\mathrm{L}}}{\partial y}-\mathrm{u}_{\mathrm{L}} \frac{\partial w^{\prime}}{\partial z}-w^{\prime} \frac{\partial W_{\mathrm{L}}}{\partial z}\right] \\
+\mu \frac{\partial}{\partial x}\left[\mathrm{~V}_{\mathrm{L}} \frac{\partial u^{\prime}}{\partial y}+v^{\prime} \frac{\partial \mathrm{U}_{\mathrm{L}}}{\partial y}+\mathrm{V}_{\mathrm{L}} \frac{\partial v^{\prime}}{\partial x}+v^{\prime} \frac{\partial \mathrm{V}_{\mathrm{L}}}{\partial x}\right] \\
+\mu \frac{\partial}{\partial x}\left[W_{\mathrm{L}} \frac{\partial u^{\prime}}{\partial z}+w^{\prime} \frac{\partial \mathrm{U}_{\mathrm{L}}}{\partial z}+W_{\mathrm{L}} \frac{\partial w^{\prime}}{\partial x}+w^{\prime} \frac{\partial W_{\mathrm{L}}}{\partial x}\right]
\end{array}
$$

which is the linearized form whose solution is desired. If the viscosity is not constant, the explicitly linearized form becomes more complicated.

The effect of the turbulence model on the perturbation field is an important consideration and has been examined in the literature. In the context of RANS, Crouch et al. [30], show that accurate global stability analyses can be obtained if the perturbation includes the effect of turbulence - in their case the Spalart-Allmaras model. If an explicit linearization is to be carried out, the turbulence closure must therefore be considered. For $L E S$, turbulence closure is incorporated with implicit [37] or explicit [38] sub-grid models. The former have been very successful [39, 40] but are derived from the mesh and the algorithm whose form is not explicitly known, while the latter require evaluation of several quantities that depend on the local flow variables. Linearization is then a non-trivial effort and must be performed separately for each model used. In the case of the synchronized LES method, since the baseline and twin simulations have the same sub-grid model, linearization is implicit and only requires that the value of $\epsilon$ be small enough, as determined from simple tests. The difficult task of explicit linearization is thus avoided with the current approach.

The procedure is also relatively straightforward to implement into existing $L E S$ codes. There are many possibilities. The one chosen by the authors is to define three Message Passing Interface (MPI) communicators. The first is global (MPI-215 COMM_WORLD say) and addresses both baseline and twin for use in communicating parameters such as Mach and Reynolds numbers, meshes and time-step sizes. The second (MPI_COMM_INDIVIDUAL) encompasses processors in each simulation (baseline or twin) and is employed to specify boundary conditions and other operations that do not affect the other computation. The final communicator (MPI_COMM_CORRESPONDING) identifies for each processor in the baseline, its corresponding processor in the twin (and vice versa). This communicator can be employed to either 
transfer the forcing (if native) or to compute the perturbation which is the difference between the twin and the baseline.

With regard to boundary conditions, both baseline and twin apply the same conditions, and in general, there is no need to perform special operations for the perturbations. Under certain conditions however, the effect of forcing can influence highly unstable parts of the domain, such as thin laminar shear layer regions, where the fluctuation itself is small. The rapid amplification of disturbances in such regions can quickly engender non-linear effects. A straightforward approach to control this unde-

230 sirable growth is to limit the amplitude of the perturbations in the twin with a damping factor.

Finally, we note that although the method effectively solves a linearized system, this does not imply the perturbations themselves track only linear phenomena. The coefficients of the linearized equations are turbulent flow variables, and incorporate non-linear mechanisms. This is manifested in the characteristics of the perturbation field, whose local spectrum changes from point to point. The check for linearity, with small $\epsilon$, simply ensures that the interaction of the perturbation field with itself is negligible.

\section{Application to Mach 1.3 jet}

The procedure is now applied to a perfectly expanded Mach 1.3 jet. The baseline is the LES of the unmodified jet. The twin is another LES of the same jet, but with a forcing term in the Navier-Stokes equations limited to a chosen spatial window. The difference between the two is the perturbation, which is the solution of the linearized Navier-Stokes equations. The forcing is the same as for the twin, 245 and the linearization is around the evolving instantaneous baseline state. We describe the $L E S$ method used for the baseline and twin simulations, the forcing regions examined, the properties of the synchronized LES and finally, the effect of turbulence in the chosen forcing regions on the near-acoustic field.

\subsection{LES method and baseline simulation}

250 The LES approach is the same as that successfully employed in Gaitonde and Samimy [41] and Speth and Gaitonde [42], which describe the scheme in detail. Briefly, the governing equations are the full 3-D, unsteady, compressible Navier-Stokes equations written in curvilinear coordinates. Both baseline and twin have the same form, except for the forcing in the latter. The reconstructed flow variables are filtered using the van Leer harmonic limiter [43], which effectively dampens out mesh-related high frequency oscillations as shown in Gaitonde and Samimy [41]. The flux is obtained with the third-order upwind-biased Roe scheme [44]. Time integration is achieved through an implicit second-order diagonalized [45] Beam-Warming approximate factorization method [46].

260 The parameters mimic the experiments of Samimy et al. [47]. Thus, the nozzle exit diameter is $\mathrm{D}=0.0254 \mathrm{~m}$. At the nozzle exit, the velocity is $\mathrm{U}_{\text {jet }}=391 \mathrm{~m} / \mathrm{s}$, the density is $\rho_{\text {jet }}=1.576 \mathrm{~kg} / \mathrm{m}^{3}$ and the temperature is $T_{\text {jet }}=224 \mathrm{~K}$. The ambient air temperature is $T_{\infty}=300 \mathrm{~K}$. All flow variables are non-dimensionalized with the 


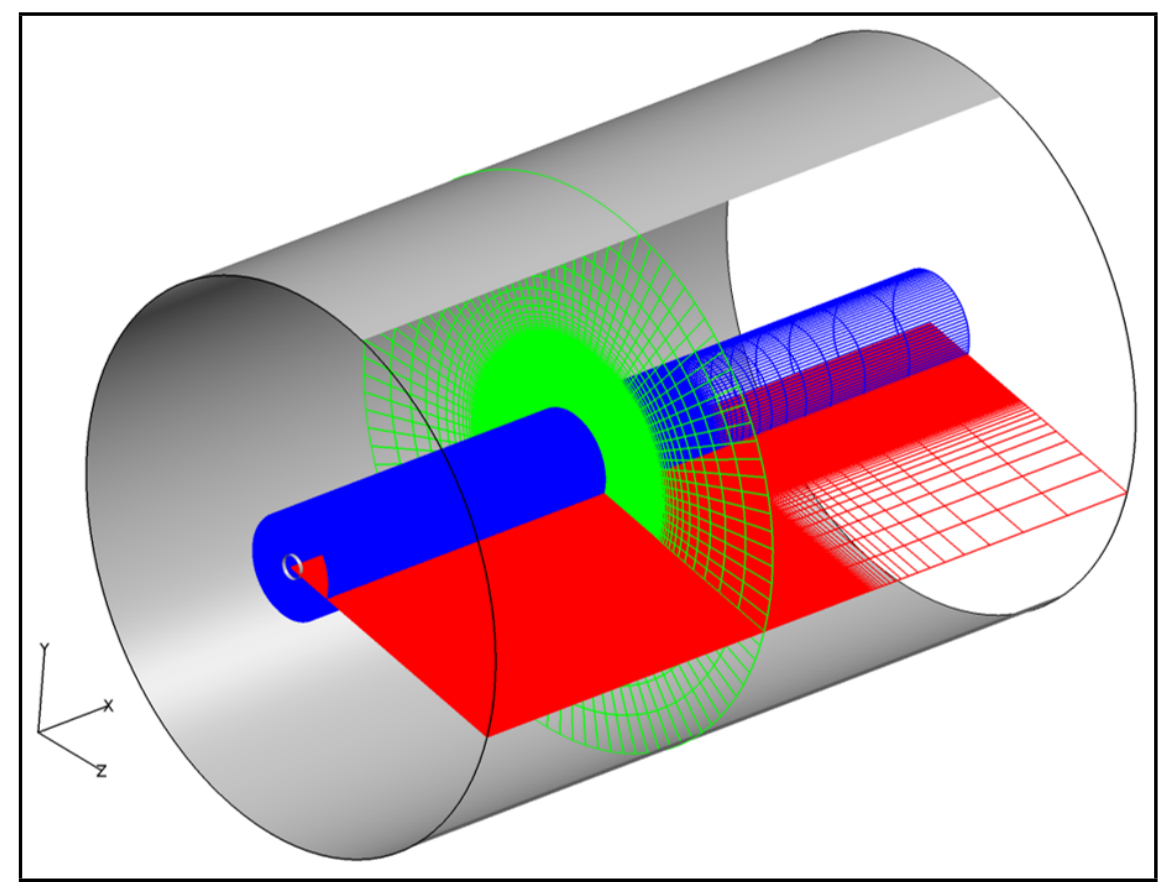

Figure 2: Computational grid employed for the LES. The nozzle sleeve is visible on the left face of the cylindrical mesh outline. Three mesh surfaces at constant $x, r$ and $\theta$ are shown.

nozzle exit conditions. This results in a Reynolds number of $1.1 \times 10^{6}$ based on $\mathrm{D}$ and a characteristic time, $\mathrm{T}_{\mathrm{c}}=\mathrm{D} / \mathrm{U}_{\text {jet }}$ of $6.496 \times 10^{-5} \mathrm{~s}$. The non-dimensionalized time-step-size is $\Delta t / T_{c}=0.001$.

A structured cylindrical mesh is employed, as shown in Figure 2. In the radial direction, the grid is refined near the centerline and lipline while clustering is employed in the axial direction near the nozzle. Gradual mesh stretching away from the jet ensures the adequacy of characteristic farfield boundary conditions. The computational domain extends 30 and 10 jet diameters in the axial and radial directions respectively. The inlet velocity is specified on the boundary-face within the nozzle region. Characteristic boundary conditions are enforced on the end-caps and outer-surface of the cylindrical domain. In the azimuthal direction, an overlap of 5 points is used to introduce a branch cut and variables are ensured to be single-valued by equating two points on the edge of the boundary with corresponding interior points on the other side of the cut. The centerline is at $r=0$ and this singularity is treated as a boundary condition by ensuring solution continuity.

Detailed comparisons with experimental data of Samimy et al. [47], including cen280 terline mean and fluctuating streamwise velocities as well as nearfield pressure probe measurements are presented in [41] and [42]. Select results from Gaitonde and Samimy [41] are shown in Figure 3. Two meshes are employed. Grid 1 is comprised of $422 \times 242 \times 105$ points along the streamwise, radial and azimuthal directions respec- 


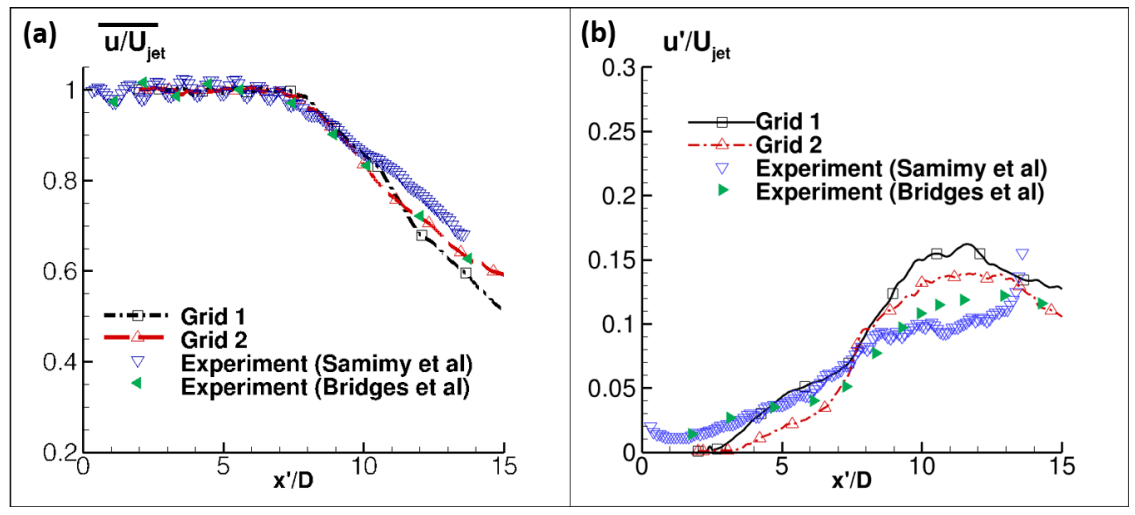

Figure 3: Comparison of computed results on jet-axis with experiments of Samimy et al. [47 and Bridges and Wernet [48]: (a) mean streamwise velocity component and (b) rms value of fluctuating streamwise velocity component, $u^{\prime}$. Figure reproduced from Gaitonde and Samimy [41]

tively, while Grid 2 consists of $580 \times 361 \times 161$ points. Computed mean (Figure 3 a) and fluctuating rms (Figure 3 p) streamwise velocities along the centerline are shown together with experiments of Samimy et al. [47] as well as other experimental data published by Bridges and Wernet [48] at approximately the same parameters. When the procedure of Bodony and Lele [49] is applied to match the core length, the decay rates downstream are captured accurately. The rms of the fluctuating streamwise component, Figure 3 b), shows the improved resolution of smaller scales in the finer mesh, reducing the effect of implicit sub-grid scale model. Overall, the comparison with experimental data is found to be satisfactory and within the limits reported by other studies [50, 49] and thus, Grid 1 is used for the synchronized LES simulations below. One of the reasons for the good agreement with experimental data is that the boundary layer exiting the nozzle is known to be very thin [47] as discussed in Speth and Gaitonde [42] and the assumption of a uniform flow at the inlet of the nozzle sleeve is sufficient.

The jet becomes turbulent relatively quickly downstream of the nozzle exit, to yield a rich variety of structures. Some of these are visualized in Figure 4 with the Q-criterion [51]. Hairpin-like coherent structures are clearly observed. With time, these propagate downstream, interacting with each other to generate the various phenomena observed, including entrainment and noise. Figure 4 (b) shows details of these structures, each of which is comprised of a tip (or head), in the outer lower speed region with legs protruding towards the center. The forcing is introduced into this evolving highly turbulent flow.

\subsection{Forcing regions of interest}

The acoustic signature is dependent on all turbulent regions in the jet. However, numerous efforts correlating farfield noise with different regions of the jet have identified high correlation values with the region after the core collapse near the centerline [21]. To illustrate the T-LNS procedure, we use native forcing, i.e., boost the existing 


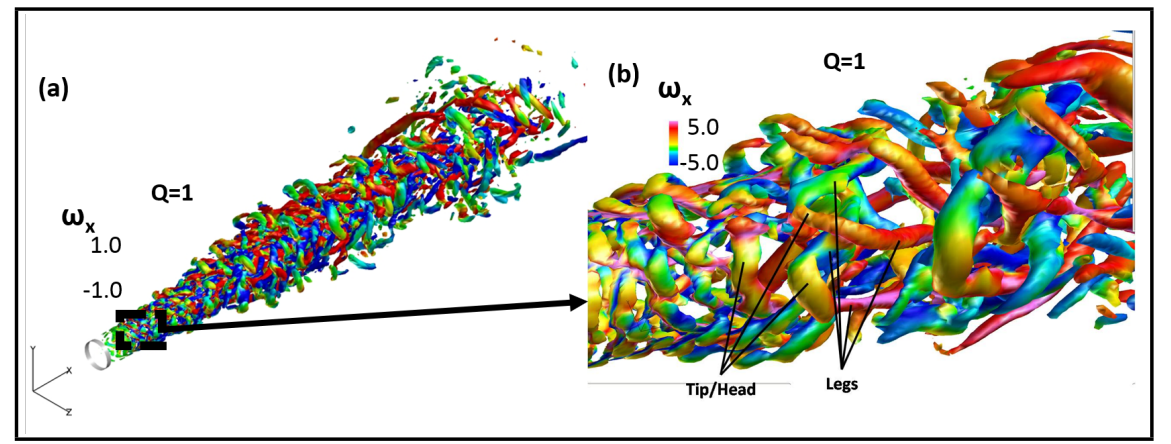

Figure 4: (a) Instantaneous flowfield of the jet visualized through the Q-criterion colored by streamwise vorticity. (b) Detailed visualization of the downstream moving structures indicating hairpin vortices.

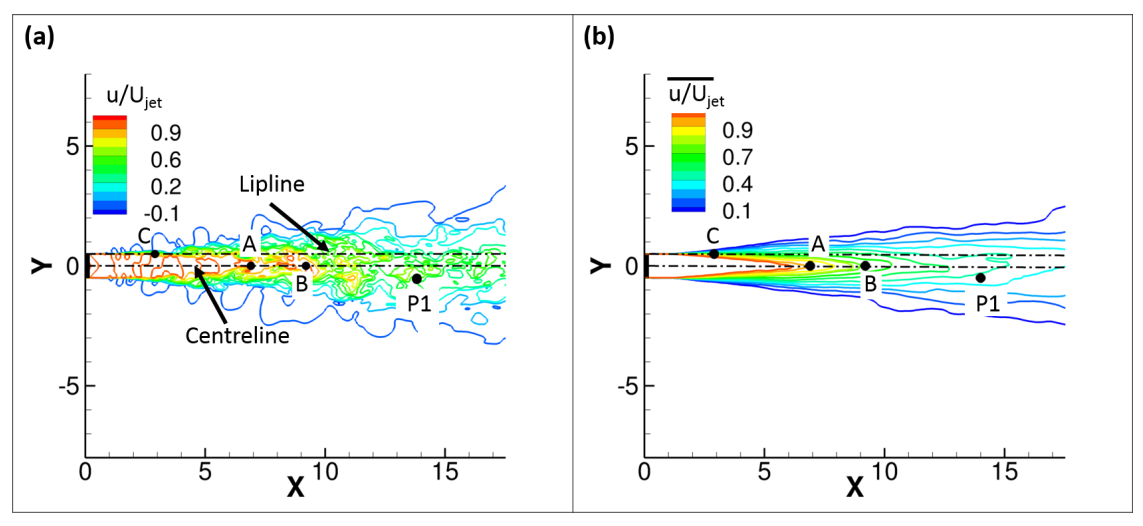

Figure 5: (a) Instantaneous and (b) mean streamwise velocity contours of the baseline simulation. Forcing regions examined (A, B and $\mathrm{C}$ ) are indicated along with sampling point to monitor the perturbation, $P 1$

fluctuation, in several regions along the axis and the lipline of the twin. The results are condensed by considering three distinct forcing regions. These are shown in Figure 5 in the context of instantaneous and mean streamwise velocity contours. Five cases are considered, referred to as Cases A, B, C, AB and AC. Case A introduces the forcing at location A, near the end of the potential core on the centerline of the jet (see Table 1 for detailed coordinates). Case B also applies the forcing on the centerline of the jet, but downstream of the collapsed core. Case $\mathrm{C}$ forcing is located near the nozzle exit on the lipline. To explore superposition and linearity, Case AB simultaneously introduces forcing at both $\mathrm{A}$ and $\mathrm{B}$. To study the interaction of two very dissimilar forcing locations, Case AC considers forcing at A and $\mathrm{C}$ together. These primary forcing regions are all point locations, chosen to limit the spatial extent to highlight specific localization of acoustic sources. Larger regions can be used if necessary, though the interpretation of the results may be difficult at sizes larger than local integral length-scales, since the region may then encompass different noise producing mechanisms that blend together. To address the effect of source dimensions, a dif- 
Table 1: Coordinates of forcing locations

\begin{tabular}{ll}
\hline Case & Source \\
\hline A & Point A $(6.897,0,0)$ \\
B & Point B $(9.242,0,0)$ \\
C & Point C $(2.988,0.5,0)$ \\
AB & Points A and B \\
AC & Points A and C \\
\hline
\end{tabular}

fuse source is considered in Section 3.5 by extending the forcing window of Case A by a small region. Additional variant simulations designed to examine the effect of transients are introduced in context below. In addition to planar data, Point $P 1$ (Figure 5), located at $x / D=14, y / D=-0.5$ (lipline) is employed as a probe for some of the 330 discussion. Also, although the perturbation of all quantities is computed, for analysis, we primarily investigate the pressure perturbation field. Other variables such as the velocity components yield similar conclusions and are not shown.

Since the forcing is derived from native fluctuations, they contain all resolved physical scales in the flow, including fine-scale turbulence as well as larger coherent features. As an example, the spectral content of the native signal for Case A is analyzed using Empirical Mode Decomposition (EMD). EMD is a data-driven approach that decomposes signals into modes, and has proven insightful in analyzing turbulent data[52]. Through a process called sifting, EMD splits the signal into its component modes, denoted as Intrinsic Mode Functions (IMFs). Figure 6 shows 340 the component scales in the forcing signal for Case A. The full pressure-signal is shown in Figure 6(a) versus non-dimensional time. Its power spectral density $(P S D)$, shown in Figure 6 d) as PSD-Signal, is broadband as expected, since it is near the fully-turbulent core collapse location. $P S D$ is plotted with the nondimensional frequency in terms of Strouhal number $(S t)$, defined as $S t=\mathrm{fDU}_{\mathrm{jet}}^{-1}$, where $f$ is the frequency in $\mathrm{Hz}$. The sum of the first three IMFs of this signal obtained through EMD is shown in Figure 6(b). These indicate the smallest component scales (or high frequency, HF) in the signal. The $P S D$ of the HF component is shown in Figure 6 d) as PSD-HF: it comprises most of the energy above $S t=1$. This is considered as the random part of the signal since coherent features in jets are usually observed below $S t=1$. The coherent part of the signal is shown in Figure 6(c) as LF which is obtained by subtracting the high-frequency component from the total signal. The spectrum in Figure 6 d), PSD-LF, populates the energy levels below $S t=1$ and can be considered to be the contribution of the large-scale structures in the flow. Although we have considered the complete native signal in

355 this work, one could, if desired, examine the filtering properties of the jet to HF and LF components separately. Similar theoretical studies of the combined effects of the mean, coherent and fine-scale motions on turbulent jets are presented in Mankbadi [53] and Mankbadi [54].

The synchronized LES procedure thus clarifies how local disturbances are filtered by turbulence in the rest of the jet to synthesize the near acoustic field. The technique will provide information on sound directivity and farfield signa- 

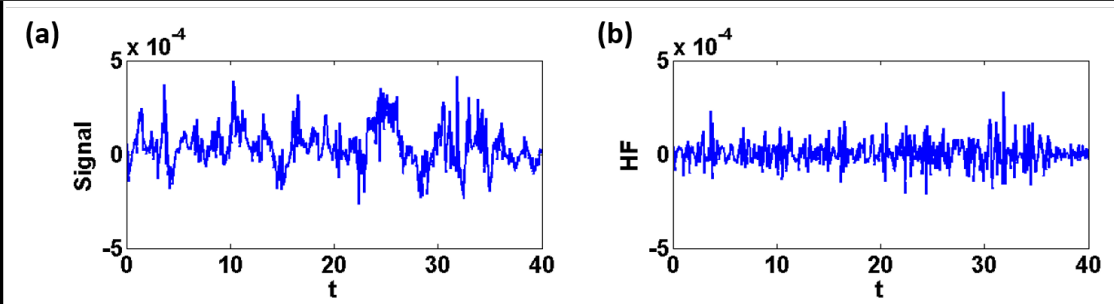

(c)
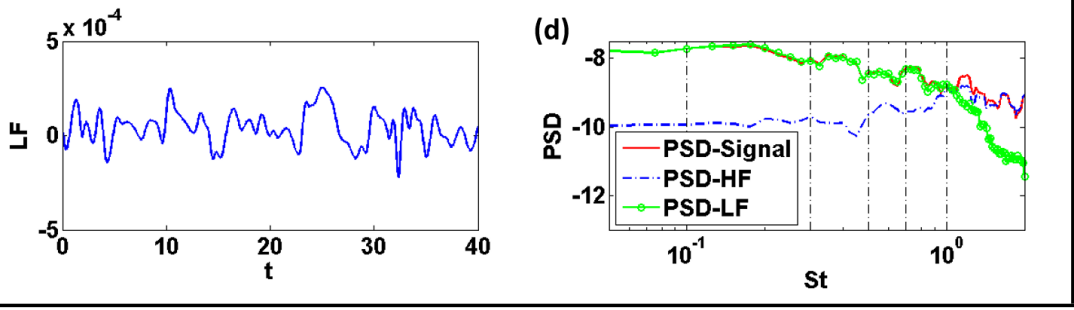

Figure 6: (a) Pressure component of forcing signal in Case A, (b) High-frequency components of signal in (a), (c) Coherent component of signal in (a), (d) PSDs of the above mentioned three signals.

ture and has the advantage that it (a) avoids simplifications associated with mean (averaged) basic states, and (b) allows the effect of specific regions of interest to be isolated without depending on post facto correlations that can be ambiguous in terms of causation.

\subsection{Instantaneous features of perturbation field}

In this section, we highlight the qualitative aspects of the perturbation field obtained with each of the forcing cases. To illustrate the basic properties, Figure 7 shows results ten characteristic times after the start of forcing for Case A. Figures 7 (a) and (b) show

370 Q-criterion iso-levels of the baseline and twin simulations. At this level, they appear identical, since the forcing amplitude is kept small by the scaling factor $\left(\epsilon=10^{-6}\right)$. However, their difference, shown in Figure 7(c) on the vertical plane, clearly shows the desired perturbation field at that instant. Near the marked source location and downstream of it, perturbation values are relatively high. Away from the axis however, the evolution into well-defined wave patterns is clearly evident. These are now studied in greater detail for each forcing region.

Although the main insights are obtained by statistical analysis of the forcing (Section 3.5p, qualitative features of the unsteady perturbation propagation provide significant insight into the filtering properties of the jet. Figure 8 shows the instantaneous perturbation field of Case A at the noted non-dimensional time instants. The forcing location is again marked in Figure 8 (a) and Figure 8 (b). In the initial phase of development (Figure 8(a)), wavefronts are observed to propagate primarily in the downstream and sideline directions. Upstream propagation is inhibited because of the supersonic core. At later time instances, Figure 8 (b), the downstream traveling perturbations am385 plify after the end of the potential core. We note in this context consistency with some observations based on wavepacket models by Cavalieri et al. [55], which successfully model the manner in which temporal amplification localized in space and 

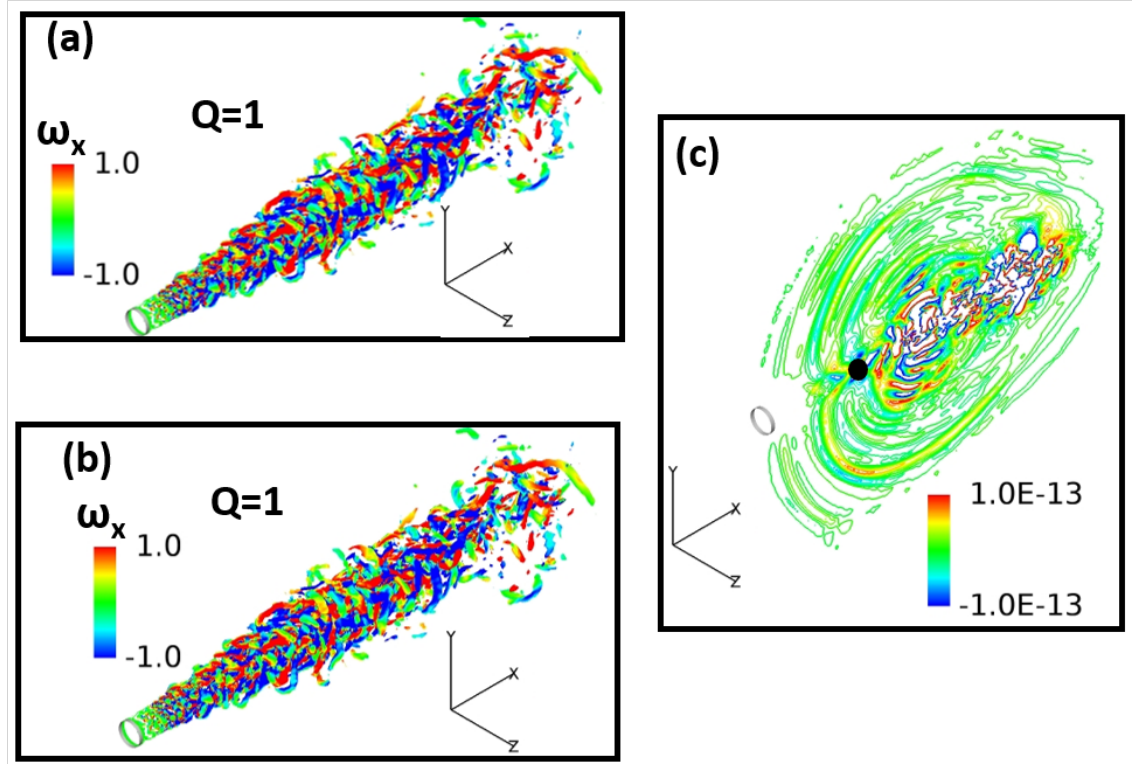

Figure 7: Instantaneous flowfield of the baseline (a) and twin (b) simulations visualized through the Qcriterion colored by streamwise vorticity. (c) Difference in pressure of the twin and the baseline on a vertical plane through the axis indicating the propagated perturbation field. The dot indicates the forcing location.

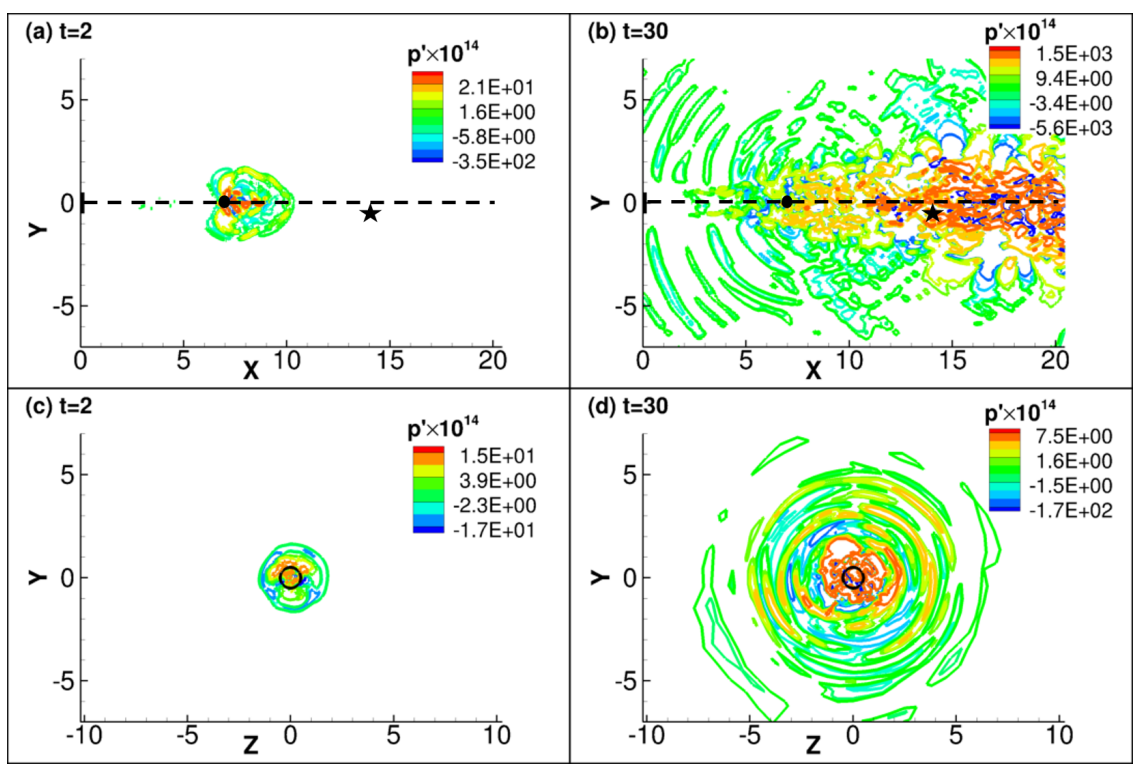

Figure 8: Scaled pressure perturbation field in Case A at the indicated non-dimensional time instants. (a) and (b) show the contours on the $Z=0$ plane and (c) and (d) indicate the same on a cross-flow plane at the forcing location. The forcing location is marked by the dot on the centerline of the jet. The stars in (a) and (b) represent point $P 1$ where the time trace of the perturbation field will be analyzed. 


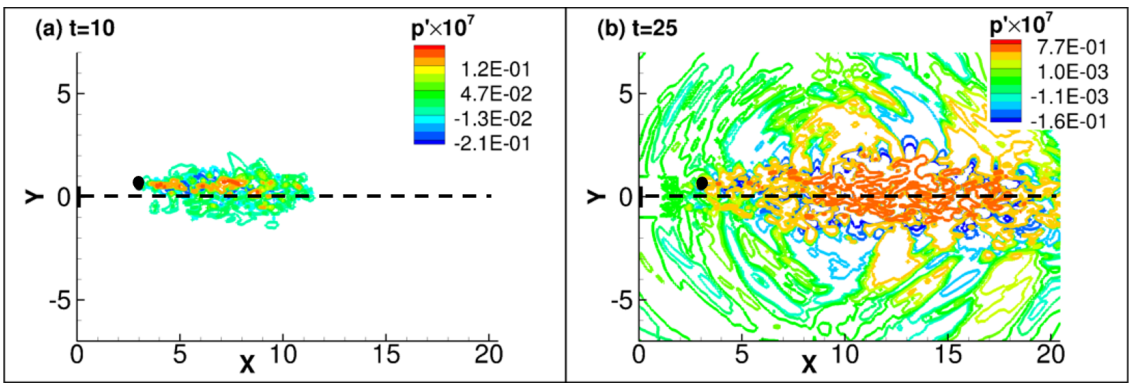

Figure 9: Scaled pressure perturbation field for Case $\mathrm{C}$ at the indicated non-dimensional time instants on the $Z=0$ plane. The forcing location is marked by the dot above the centerline of the jet.

time leads to high amplitude downstream radiation. These dynamics were found to be most prominent near the end of the potential core. The present results show similar intermittent amplification of the perturbation field, particularly after the core collapse location. After this amplification region, the perturbations propagate as though they are subjected to secondary sources, with a strong component along low aft angles to the axis.

Figures 8 (c) and (d) show the perturbation field along a cross-flow plane containing the forcing region at the same time instants. The perturbation has no clear structure near the axis, since the flow there is turbulent. Moving outwards however, azimuthally coherent features and recurring intermittent wave fronts are observed, but the jet remains axisymmetric in a statistical sense. This intermittency is a result of the filtering effect of the unsteady jet, and is an important factor in jet noise [56]. Case B, which 400 has a centerline source further downstream, also produces a similar perturbation field and is not shown for brevity.

Figure 9 shows the instantaneous perturbation field for Case $\mathrm{C}$, in which the source is located on the lipline of the jet. Note that the time instants are different from those employed for Case A, and are chosen to highlight primary features of Case C. Figure 9 (a) shows that the perturbation field tracks the roll-up and evolution of shear layer instabilities, which manifest as vortical structures. The perturbations are essentially first channeled towards the potential core end region on the centerline, where they are again amplified to a higher level. Perturbation levels (for the same $\epsilon=10^{-6}$ ) are four to five orders of magnitude higher than in the centerline cases. With increasing time, 410 Figure 9 (b), the perturbation travels downstream. In addition to intermittent wavefronts at low angles which are qualitatively similar to those observed for Cases A and B, relatively stronger and well preserved upstream propagating wavefronts are also visible. This highlights the contribution of initial shear-layer transition effects to upstream propagating signals in the jet.

${ }_{415}$ The above results indicate that all cases experience perturbation amplification in the downstream regions between 10 to 20 jet diameters. This region of strong amplification eventually results in highly directional downstream perturbations. Such extended axial sources resulting in high correlations with shallow downstream angles have been reported in experiments by Viswanathan et al. [57]. In round jets excited by a combi- 


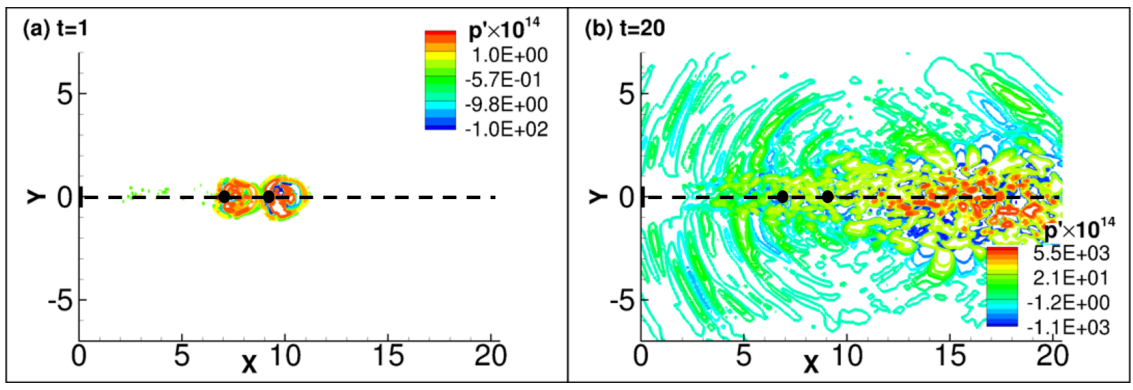

Figure 10: Scaled pressure perturbation field in Case $\mathrm{AB}$ at the indicated non-dimensional time instants on the $Z=0$ plane. The forcing locations are marked by dots on the centerline of the jet.

\subsection{Properties of the synchronized LES method}

We first explore the linearity aspect by considering Cases A, B and AB. Figure 12 (a) shows the perturbation field at Point $P 1$ (see Figure 5) due to Case A and 450 Case B. Each has an initial time range where no disturbance is observed - this reflects 


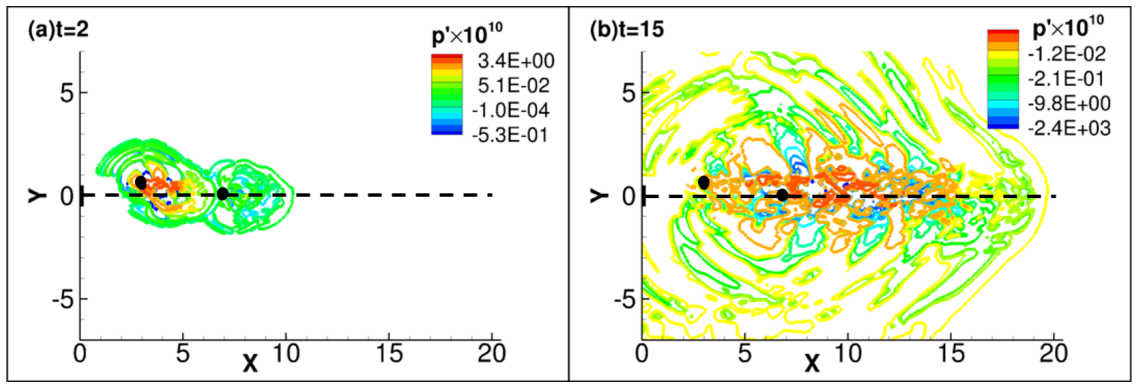

Figure 11: Scaled pressure perturbation field in Case $\mathrm{AC}$ at the indicated non-dimensional time instants on the $Z=0$ plane. Forcing locations are marked by the dots on and above the centerline of the jet.

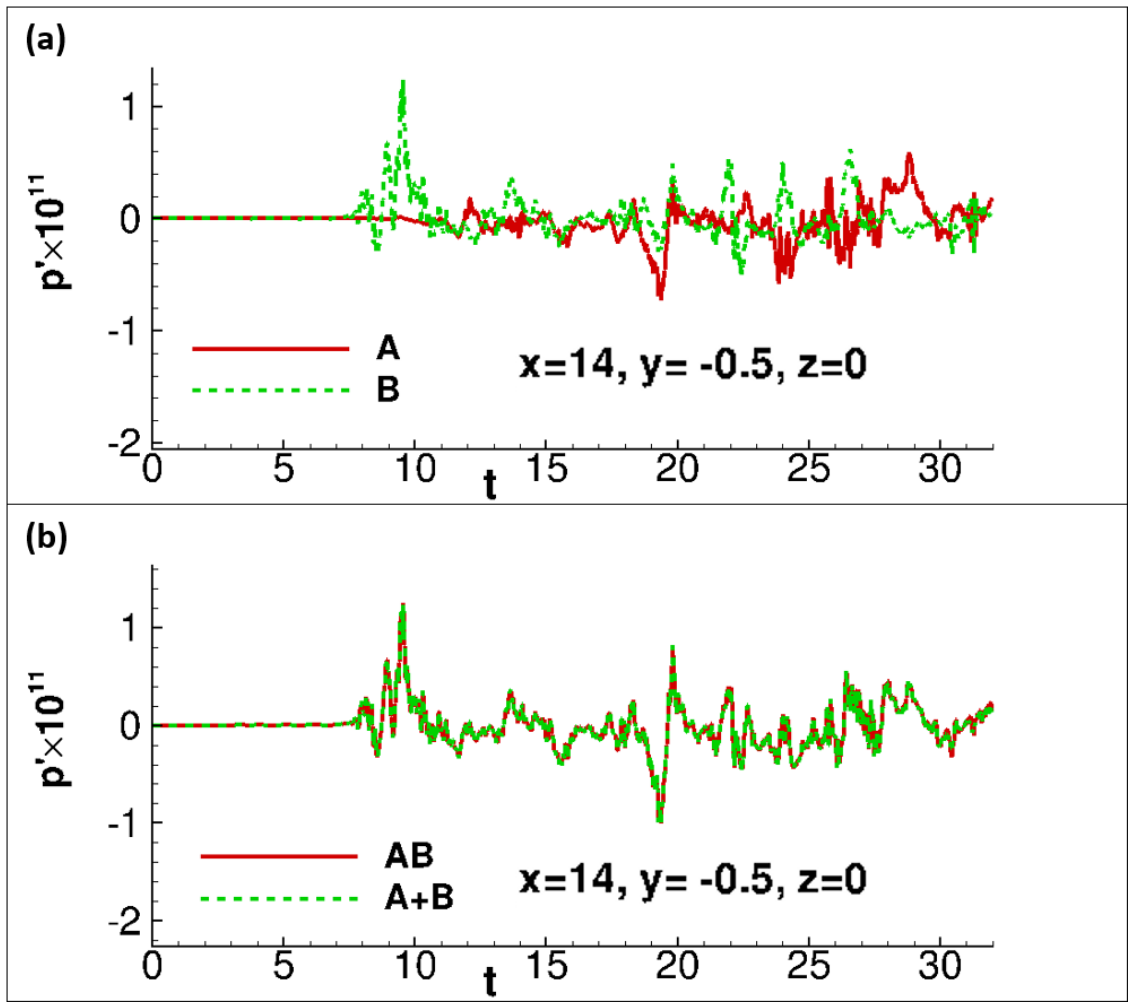

Figure 12: Scaled pressure perturbation test for linearity at Point $P 1$. (a) Time trace of Case A and Case B. (b) Sum of Cases A and B with the perturbation of Case AB. 


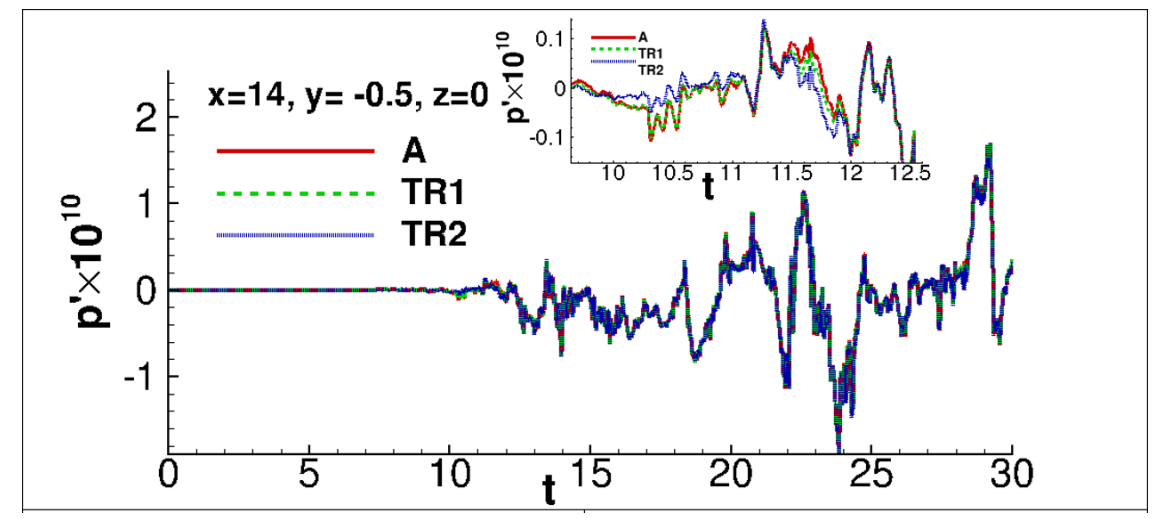

Figure 13: Scaled pressure perturbation at Point $P 1$ The curve denoted $\mathrm{A}$ is the time trace without ramping, while TR1 and TR2 are time traces when the forcing is gradually ramped up over characteristic times of $\mathrm{T}_{\mathrm{c}}=0.5$ and $\mathrm{T}_{\mathrm{c}}=1$ respectively. The inset shows the same curves, but is zoomed in to highlight the differences in them.

the time required for the effect of forcing to reach point $P 1$. Subsequently, although the perturbation fields of the two forcings have same order of magnitude effects on the amplitude, their spectral content is very different. This follows from the fact that they propagate through a different turbulent environment. Figure 12 b) shows the addition of the two signals of Figure 12 a) (denoted A+B), together with the response obtained from Case AB. The two curves are indistinguishable, indicating that the chosen perturbation levels are small enough that linearity holds. Results with $\epsilon$ ranging from $10^{-3}$ to $10^{-9}$ indicate the same spectral content in each signal, though the amplitude scales linearly with $\epsilon$.

In the process of post-processing DNS data, Freund [59] notes the possible effect of finite time-length of the signals when using Fourier transforms. A related question is whether the perturbation requires an initial establishment time. To evaluate this, the forcing was ramped up by gradually increasing the value of $\epsilon$ using the functional form:

$$
\epsilon(t)=0.5 \epsilon_{\max }\left\{1+\tanh \left[\frac{6 \mathrm{t}}{\mathrm{T}_{\mathrm{p}}}-\mathrm{t}_{\mathrm{o}}\right]\right\}
$$

where $t$ is the time, $t_{o}=3$ and $\epsilon_{\max }=10^{-6}$. Figure 13 shows the pressure perturbation at point $P 1$ for Case A with two different ramp-up times $\mathrm{T}_{\mathrm{p}}$ of $0.5 \mathrm{~T}_{\mathrm{c}}$ (TR1) and $1.0 \mathrm{~T}_{\mathrm{c}}$ (TR2) respectively. Other than the initial variation highlighted in the corresponding inset, the three signals achieve the same amplitude and spectral content in about $2.5 \mathrm{~T}_{\mathrm{c}}$. Thus, the fact that the forcing is native and is injected into a statistically stationary baseline jet results in relatively short transient effects. The sensitivity of the results to size and edges of the forcing region is discussed below.

\subsection{Statistical analysis of nearfield perturbation patterns}

The intermittency observed in Figs. 8 and 9 is further analyzed quantitatively using spatio-temporal correlations. We seek to use the results to understand how the forcing region influences the nearfield. For this, we adopt a forward correlation approach 


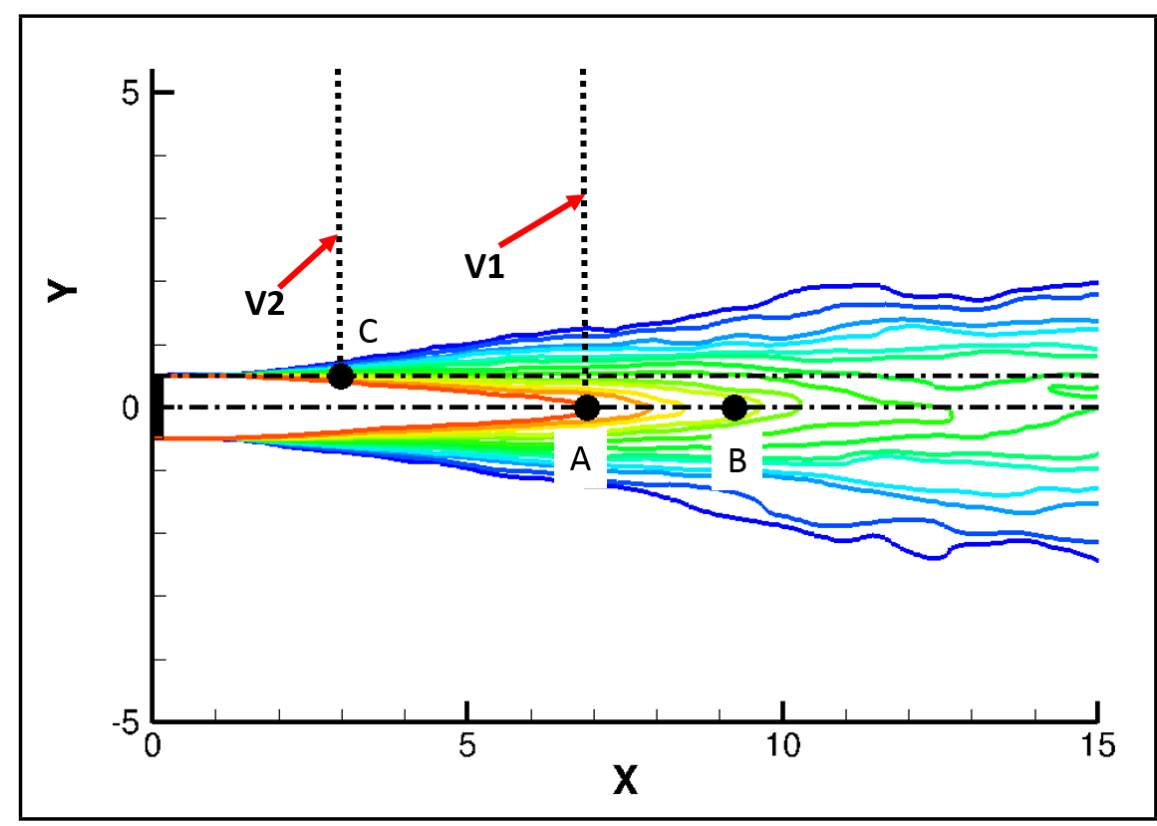

Figure 14: Lines along which pressure perturbation is obtained for various cases for statistical analysis. VI and $V 2$ are vertical lines directly above the forcing locations for Cases $\mathrm{A}$ and $\mathrm{C}$ respectively.

in which the forcing signal is correlated with its own induced nearfield perturbation. Specifically, the perturbation-boost at the forcing location (which, to reiterate, is the scaled fluctuation, see Eqn. 2p is correlated with each point in the domain and the correlation constant (denoted cc) is obtained as a function of time lag.

We first consider $1-D$ correlations. Figure 14 shows two vertical lines V1 and V2 above points $\mathrm{A}$ and $\mathrm{C}$ respectively. The cc contours correlating the forcing with the perturbation signals along the points of these lines for Cases A and $\mathrm{C}$ respectively are shown in Figure 15. The abscissa is the time lag, while the ordinate is the radial distance. The correlation contours indicate the presence of inclined rays of alternating 480 sign, which represent succeeding crests and troughs of wavepackets. These patterns are the footprints of the recurring wavefronts that are generated from the turbulence in the forcing region and which propagate outwards. Each streak represents an event arising from the filtering effect of the jet. At any fixed radial location (horizontal line), the lag difference between two crests represents two consecutive events. Likewise, an event traverses two points separated by a distance $d r$ within a time gap of $d t$ as indicated in Figure 15 (a). The slope thus indicates the speed of these wave fronts, which can be approximately evaluated by knowing the reference length and time scales. The wave speed estimated in this manner is about $350 \mathrm{~m} / \mathrm{s}$ which is the approximate speed of sound in the medium.

The patterns of spatio-temporal correlations in Figure 15 b) (data obtained from line V2 in Figure 14 indicate that the length scales associated with the lipline source $($ Case $\mathrm{C})$ are relatively larger than those of the centerline cases. In addition, the patterns 


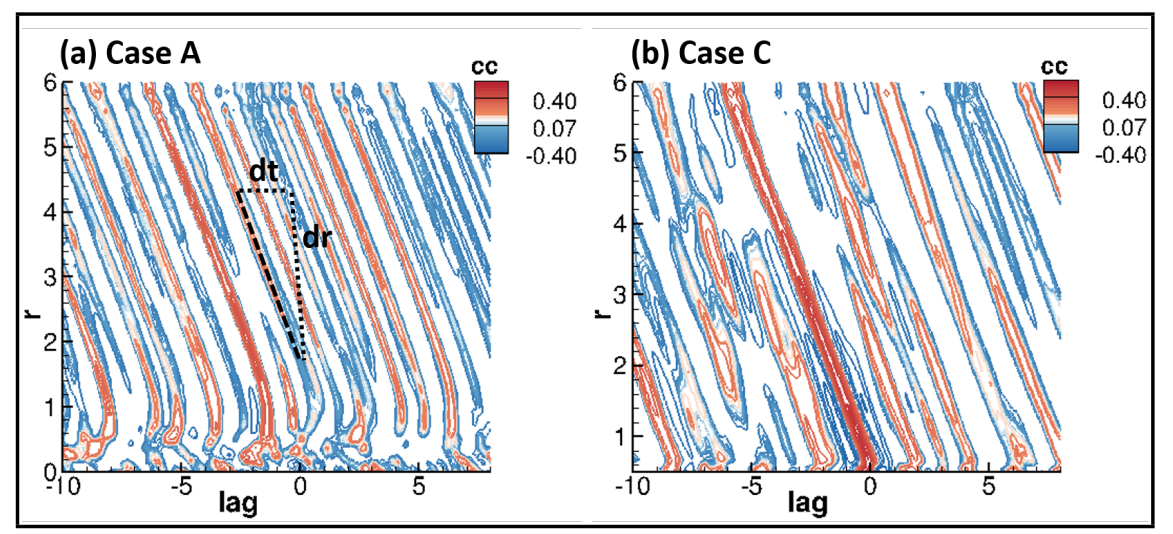

Figure 15: 1D spatio-temporal correlations for Cases A and C obtained from pressure disturbance along the vertical line $V 1$ and $V 2$ respectively. The horizontal axis represents the non-dimensionalized time lag and the vertical axis represents the non-dimensional radial distance from the jet axis. The dotted triangle in (a) represents a single event originating from core turbulence traversing a distance $d r$ within a time lag of $d t$.

are wider and less coherent. This reflects the fact that perturbations emanating from the shear layer are inherently different from those originating in the core. In particular, due to the amplification of shear layer instabilities, coherent structures are produced along the lipline which break up and create wavefronts with larger spatial extent. These larger spatial scales can also be considered to be a reason for the higher "gain" associated with lipline perturbations observed in Figure9. The optimal-frequency excitation study in $[60]$ revealed that the larger scales (with their associated lower frequencies) have longer lifespan in the streamwise direction. Hence they are more efficient than high-frequency waves in pumping energy from the mean flow, which in our case is actually the time-varying baseline flow. The correlation rays in the centerline case appear more distinct, indicating that the propagated intermittent wavefronts have lesser interaction with each other. The discontinuities in perturbation-correlation 505 rays due to the lipline forcing suggests that larger wavefronts coalesce and disintegrate while being oriented towards sideline angles. Each location thus has a unique role in creating the intermittency of overall nearfield signal of the jet.

To investigate the effects of the spatial extent of the forcing region on the statistical properties of the nearfield, a $1-D$ correlation analysis is performed with a diffuse region around location $\mathrm{A}$, by enlarging the window and allowing $\mathscr{W}(\mathbf{X}, t)$ to decay near the boundaries. The window function is unity at location $\mathrm{A}$ and decays linearly to zero within 0.2 jet diameters in the axial and radial directions. There is no azimuthal variation in the source strength. The diffuse source-region has to be kept sufficiently small if meaningful comparisons are to be made with the cor15 responding point-forcing case. As in Case $\mathbf{A}$, the forcing here is also continuous in time. The results, Figure 16, show that the diffuse forcing region yields a perturbation field similar to that observed in Figure 15(a) in terms of the slope of the correlation rays. The diffuse source shows a slightly denser distribution of the wavefronts near zero lag value. This is expected as it reflects the contribution of the slightly larger 


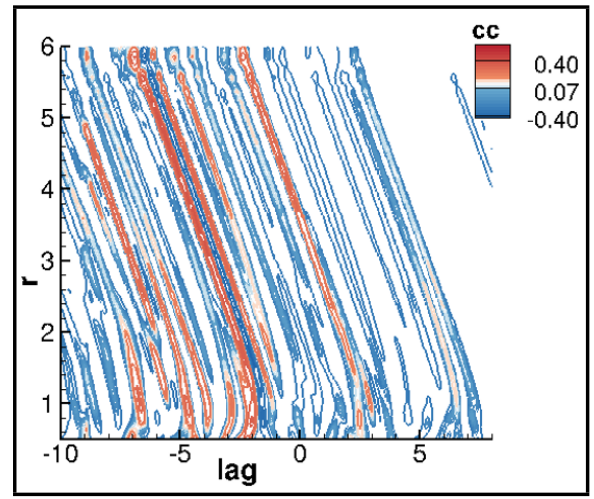

Figure 16: 1D spatio-temporal correlations for diffuse forcing at $\mathrm{A}$ obtained from pressure perturbation signals along the vertical line $V 1$. The horizontal axis represents the non-dimensionalized time lag and the vertical axis represents the non-dimensional radial distance from the jet axis.

forcing region.

The above analysis has only examined correlations along the vertical direction, which correspond to the general direction of propagation of lower amplitude higher frequency noise. It is well known however, see e.g., Tam et al. [19] that the acoustic field has strong directionality, with higher amplitude waves propagating at shallow angles to the axis. To evaluate correlations in other directions, the spatio-temporal correlation analysis is extended to $2-D$. Thus, the correlation is computed between the scaled fluctuation (perturbation boost) at the forcing location and perturbation signal at every other point on the $Z=0$ plane, to yield a $2-D$ snapshot of $c c$ at each lag value. As an example, the $c c$ contour at zero lag value is shown in Figure 17 for Case A. Figure 17(a), along the streamwise plane, highlights the recurring wavefronts observed earlier in Figs. 8 and 15 but provides more information. Being a correlation, unlike Figs. 8 and 15, Figure 17 a) indicates the persistence of the primary nearfield phenomena as well as its relation to the forcing. The intermittent wavepacket nature is not observed in the core of the jet where the flow is highly turbulent. However, low aft angles indicate wavefronts at larger length scales (lower frequency) generated from the turbulent core. This is consistent with nearfield observations (see e.g., Tam et al. [19]). Animations varying with the time lag (not shown) indicate well-preserved wavefronts propagating in the upstream direction (intermediate frequency). This is associated with the breakdown of the shear layer near the nozzle exit.

These wavefronts are obviously three-dimensional in nature. This is shown in Figure 17 b) by plotting $\mathrm{cc}$ contours for Case $\mathrm{A}$ on an axial plane at the forcing location. As noted earlier in the context of Figure 8 (d), the recurring wave fronts appear as concentric patterns which become more predominant towards the near-acoustic field as the core turbulence dies out. The circumferential extent of these structures can be discerned from Figure 17 b). Beyond $r=3 \mathrm{D}$, the structures extend approximately $180^{\circ}$ azimuthally. At larger radial distances, the azimuthal coherence further increases to about $270^{\circ}$. Since these contours indicate directivity in the perturbation field, an inherent feature of the jet is clearly the directional selective filtering of length scales and 


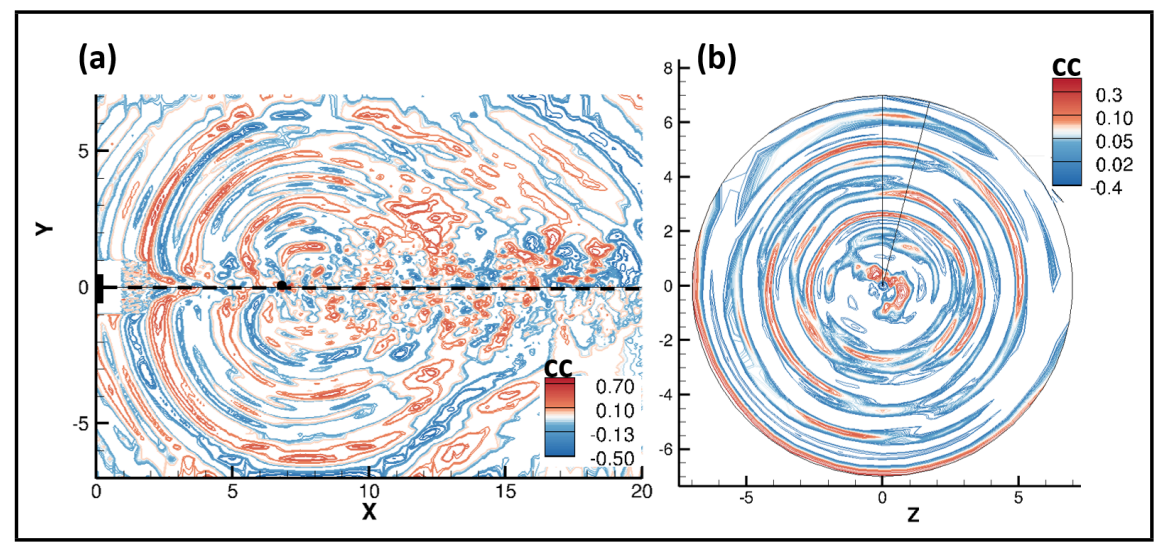

Figure 17: 2-D spatio-temporal correlations for Case A obtained from pressure perturbation signals at zero lag. (a) represents the contours on $Z=0$ plane and (b) represents the same on a cross-flow plane at the forcing location. The forcing location is marked by a dot on the centerline of the jet.

intermittency.

Figure 18 shows the corresponding $\mathrm{cc}$ contours for Case $\mathrm{C}$ to highlight the main differences associated with lipline forcing. The contours clearly demarcate the turbulent core of the jet dominated by convecting rolled-up eddies from the predominantly propagating nearfield. The events inside the core of the jet yield wavepackets of larger spatial extent than the centerline cases, as can be seen in directions perpendicular to 555 the axis near the source. In this case also, the upstream propagating wavefronts are relatively more intact and display smaller length scales. The downstream propagating perturbations show clear shallow angle directionality. Further, they are comprised of the larger spatial scales found in the flowfield.

Since the spatio-temporal features are obtained through correlation of the pressure signal, they highlight the dominant hydrodynamic features in the core of the jet. Towards the near field, the implicit filtering in the jet attenuates the hydrodynamic components and the prominent dynamics are those of the acoustic mode. The downstream acoustic radiation are associated with the coherent turbulent structures in the core.

565 The above correlation analysis has focused on the spatial aspects of the perturbation field. A measure of the temporal scales can be obtained by exploring the integral time scales of the flow. A local time scale in the flow is obtained from the autocorrelation function. For this purpose, the autocorrelation of the pressure perturbation signal is obtained at each point in the $Z=0$ plane. The lag value at which the autocorrelation 570 function first changes sign from positive to negative is taken as the local integral time scale at that location. Figure 19 shows values of local time scales of various source configurations. The $\log _{10}$ value of the time scale is plotted since it varies over several orders of magnitude. The plot then highlights the demarcation of prominent regions in the near-acoustic field. For Case A, Figure 19 a), the contours are generally symmetric 575 about the axis and clearly demarcate three different zones. Zone 3 represents the largest time scales present in the flowfield. Since the temporal and spatial scales are coupled, 


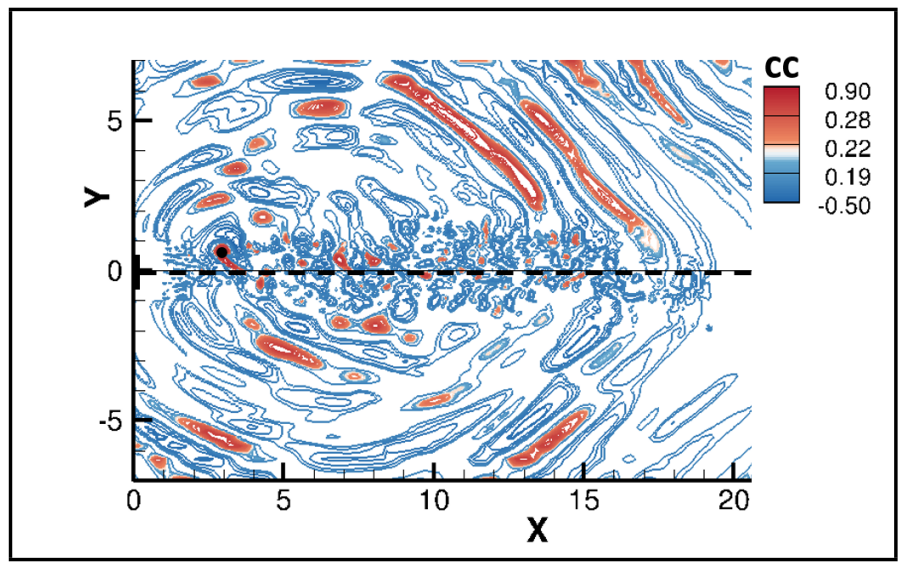

Figure 18: 2-D spatio-temporal correlations for Case $\mathrm{C}$ obtained from pressure perturbation signals at zero lag on $Z=0$ plane. The forcing location is marked by a dot above the centerline of the jet.

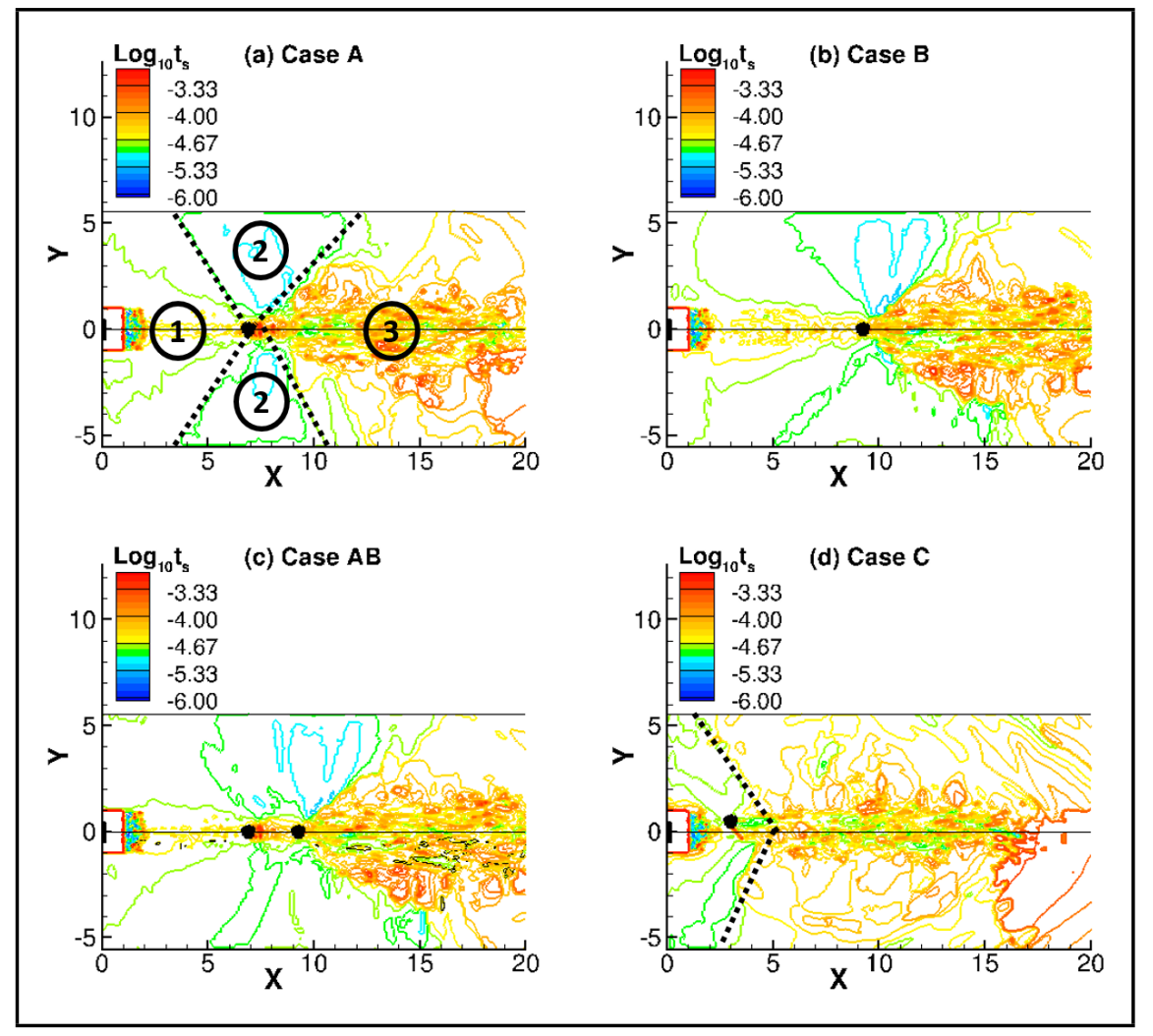

Figure 19: Local time scale of the perturbation fields in various cases. The forcing region in each case is marked by the dot. The dotted lines in (a) and (c) demarcates prominent zones in the time-scale contours. 
this zone also represents the largest scales of motion present in the flow. This corresponds to the larger wavelengths radiated at low aft angles in the downstream direction. Zone 1 corresponds to an intermediate range of time scales containing wavepackets at intermediate length scales which are well preserved and propagate upstream. Zone 2 corresponds to the smallest time scales in the flowfield. This method thus yields detailed information on how the highest frequencies are selectively guided in a direction perpendicular to the jet axis from the source location, i.e., the perturbation field provides a clear indication of the directivity induced by the core of the jet. Analysis of the total jet (not included) indicates a similar demarcation of length and time scales.

The time-scales for Case B are shown in Figure 19(b). Although the qualitative features of the time-scale distribution remain similar to Case A, the spatial extent of the highest frequencies (Zone 2) are relatively more spread out. This is likely associated with the lower velocity at the forcing location and the propagation is thus more isotropic. Figure 19.c) shows the local time scales in Case AB, where both the centerline sources are activated. As anticipated, the characteristics are a combination of those observed in Cases A and B. Case C, shown in Figure 19.d), has an inherently different forcing field, which is also reflected in the local time scale. A visible demarcation between Zones 1 and 3 is observed, but Zone 2, comprised of the highest 595 frequencies is absent. This is because of the relatively large scale structures produced in the shear layer, which emit strong perturbation wavefronts along the aft direction. Moving downstream, the time scale increases indicating that the wavelength of the emitted wavepackets increases. Although not shown, the features of the diffuse forcing case (discussed in Section 3.4 are similar to those observed in Case A, indicating that the nearfield statistics are not drastically altered by small changes (relative to the local integral scales) in forcing location.

The time-scale analysis thus provides insights into the directivity associated with specific locations where forcing is applied. The variation of magnitudes in the time-scale contours represents the distribution of the most dominant spatiotemporal scale prevalent in the flowfield. It highlights the preference of certain frequencies along different directions from the forcing location, which we interpret as directivity of jet noise. From the analysis of the different cases considered, it is clear that the centerline forcing has a different directivity compared to the lipline case.

610 Earlier studies [53], [54] have shown that the streamwise lifespan of the perturbations is inversely proportional to their wavelength. Thus high-frequency components are prevalent closer to the nozzle exit whereas the low-frequency components dominate the downstream locations. These results from axisymmetric excitation of jets indicate that the most amplified $S t$ varies nearly inversely with the axial distance. The results obtained here are qualitatively similar. The time-scale analysis in Figure 19(a) for instance, shows that there is a monotonic increase in time scales from Zone 2 to Zone 3 and this trend persists further downstream. Since time scales and frequencies are inversely related, this shows that the peak St varies inversely with the downstream axial-distance from the region of forcing. 


\section{Conclusion}

A novel approach, using synchronized Large-Eddy Simulations, has been developed and employed to examine the effect of forcing the Navier-Stokes equations linearized about the instantaneous state. The method has several advantages over explicand its time derivatives nor explicit expressions for the linearization. Furthermore, existing $L E S$ codes can be adapted with relatively minor modifications. The properties of the method, including linearity as well as the sensitivity to spatial and temporal properties of the forcing function are explored. In addition to arbitrary forcing such as white of a localized region on the surroundings. As an example of the use of the method, the problem of jet noise is considered. Native perturbations are introduced at several locations, and the results are distilled to provide insight into noise source localization. Maximum amplification is observed after the collapse of the core, leading to secondary 635 apparent sources. Perturbations on the lipline are first channeled to the centerline near the core collapse, after which the development is qualitatively similar to forcing at the core itself, but the "gain" associated with lipline forcing is much higher than those from the centerline. This results from their initial relatively rapid growth. Integral time scales indicate three different zones for the centerline forcing cases, where the source, while the lower frequencies travel along low aft angles, consistent with prior experimental and computational data.

\section{Acknowledgments}

This research was sponsored by the Office of Naval Research (Grant No: N0001413-1-0534) monitored by J. Doychak and B. Henderson, with K. Kailasanath (NRL) and J. Spyropoulos (NAVAIR) as Navy Technical Points of Contact. The simulations were performed with a grant of computer time from the DoD HPCMP DSRCs at AFRL, NAVO and ERDC, and the Ohio Supercomputer Center. The authors are also grateful to Intelligent Light for Fieldview licenses made available under their University Partnership Program.

\section{References}

[1] P. G. Drazin, W. H. Reid, Hydrodynamic stability, Cambridge university press, 2004.

[2] L. Lees, C. C. Lin, Investigation of the stability of the laminar boundary layer in a compressible fluid, NACA TN-1115 (1946).

[3] A. Michalke, The instability of free shear layers, Progress in Aerospace Sciences 12 (1972) 213-216.

[4] T. Herbert, Parabolized stability equations, Annual Review of Fluid Mechanics 29 (1997) 245-283. 
[5] M. R. Malik, Numerical methods for hypersonic boundary layer stability, Journal of Computational Physics 86 (1990) 376-413.

[6] J.-C. Robinet, Bifurcations in shock-wave/laminar-boundary-layer interaction: global instability approach, Journal of Fluid Mechanics 579 (2007) 85-112.

[7] F. Alizard, S. Cherubini, J.-C. Robinet, Sensitivity and optimal forcing response in separated boundary layer flows, Physics of Fluids 21 (2009) 064108.

[8] V. Theofilis, Global linear instability, Annual Review of Fluid Mechanics 43 (2011) 319-352.

[9] E. Touber, N. D. Sandham, Large-eddy simulation of low-frequency unsteadiness in a turbulent shock-induced separation bubble, Theoretical and Computational Fluid Dynamics 23 (2009) 79-107.

[10] P. Jordan, T. Colonius, Wave packets and turbulent jet noise, Annual Review of Fluid Mechanics 45 (2013) 173-95.

[11] A. Sinha, H. Alkandry, M. Kearney-Fischer, M. Samimy, T. Colonius, The impulse response of a high-speed jet forced with localized arc filament plasma actuators, Physics of Fluids 24 (2012) 125104.

[12] A. Sinha, D. Rodríguez, G. A. Brès, T. Colonius, Wavepacket models for supersonic jet noise, Journal of Fluid Mechanics 742 (2014) 71-95.

[13] A. Hussain, Coherent structures and turbulence, Journal of Fluid Mechanics 173 (1986) 303-356.

[14] F. Hussain, E. Stout, Self-limiting and regenerative dynamics of perturbation growth on a vortex column, Journal of Fluid Mechanics 718 (2013) 39-88.

[15] M. Lighthill, On sound generated aerodynamically: I. general theory, Proc. R. Soc. Lond. 211 (1952) 564-587.

[16] M. Lighthill, On sound generated aerodynamically: II. turbulence as a source of sound, Proc. R. Soc. Lond. 222 (1954) 1-32.

[17] C. K. Tam, K. Chen, A statistical model of turbulence in two-dimensional mixing layers, Journal of Fluid Mechanics 92 (1979) 303-326.

[18] R. Mankbadi, J. Liu, Sound generated aerodynamically revisited: large-scale structures in a turbulent jet as a source of sound, Philosophical Transactions of the Royal Society of London A: Mathematical, Physical and Engineering Sciences 311 (1984) 183-217.

[19] C. Tam, K. Viswanathan, K. Ahuja, J. Panda, The sources of jet noise: experimental evidence, Journal of Fluid Mechanics 615 (2008) 253-292.

[20] C. Bogey, O. Marsden, C. Bailly, Influence of initial turbulence level on the flow and sound fields of a subsonic jet at a diameter-based Reynolds number of 105, Journal of Fluid Mechanics 701 (2012) 352-385. 
[21] C. Bogey, C. Bailly, An analysis of the correlations between the turbulent flow and the sound pressure fields of subsonic jets, Journal of Fluid Mechanics 583 (2007) 71-97.

[34] M. Goldstein, L. S. Hultgren, Boundary-layer receptivity to long-wave freestream disturbances, Annual Review of Fluid Mechanics 21 (1989) 137-166. 
[35] B. F. Farrell, P. J. Ioannou, Stochastic forcing of the linearized Navier-Stokes equations, Physics of Fluids A: Fluid Dynamics (1989-1993) 5 (1993) 26002609.

[36] M. Choudhari, Boundary-layer receptivity due to distributed surface imperfections of a deterministic or random nature, Theoretical and Computational Fluid Dynamics 4 (1993) 101-117.

[37] F. F. Grinstein, L. G. Margolin, W. J. Rider, Implicit large eddy simulation: computing turbulent fluid dynamics, Cambridge university press, 2007.

[38] P. Moin, K. Squires, W. Cabot, S. Lee, A dynamic subgrid-scale model for compressible turbulence and scalar transport, Physics of Fluids A: Fluid Dynamics (1989-1993) 3 (1991) 2746-2757.

[39] D. J. Garmann, M. R. Visbal, P. D. Orkwis, Comparative study of implicit and subgrid-scale model large-eddy simulation techniques for low-Reynolds number airfoil applications, International journal for numerical methods in fluids 71 (2013) 1546-1565.

[40] S. Kawai, S. K. Shankar, S. K. Lele, Assessment of localized artificial diffusivity scheme for large-eddy simulation of compressible turbulent flows, Journal of Computational Physics 229 (2010) 1739-1762.

[41] D. Gaitonde, M. Samimy, Coherent structures in plasma-actuator controlled supersonic jets: Axisymmetric and mixed azimuthal modes, Physics of Fluids 23 (2011) 095104.

[42] R. Speth, D. Gaitonde, Parametric study of a Mach 1.3 cold jet excited by the flapping mode using plasma actuators, Computers and Fluids 84 (2013) 16-34.

[43] B. van Leer, Towards the Ultimate Conservation Difference Scheme V, A SecondOrder Sequel to Godunov's Method, Journal of Computational Physics 32 (1979) 101-136.

[44] P. Roe, Approximate Riemann Solvers, Parameter Vectors and Difference Schemes, Journal of Computational Physics 43 (1981) 357-372.

[45] T. Pulliam, D. Chaussee, A Diagonal Form of an Implicit ApproximateFactorization Algorithm, Journal of Computational Physics 39 (1981) 347-363.

[46] R. Beam, R. Warming, An Implicit Factored Scheme for the Compressible Navier-Stokes Equations, AIAA Journal 16 (1978) 393-402.

765 [47] M. Samimy, J.-H. Kim, J. Kastner, I. Adamovich, Y. Utkin, Active control of high-speed and high-Reynolds-number jets using plasma actuators, Journal of Fluid Mechanics 578 (2007) 305-330.

[48] J. Bridges, M. Wernet, Turbulence associated with broadband shock noise in hot jets, AIAA Paper 2008-2834 (2008). 
[49] D. Bodony, S. Lele, On using large-eddy simulation for the prediction of noise from cold and heated turbulent jets, Physics of Fluids 17 (2005) 085103.

[50] J. Kim, A. Afshari, D. Bodony, J. Freund, LES investigation of a Mach 1.3 jet with and without plasma actuators, AIAA Paper 2009-290 (2009).

[51] G. Haller, An objective definition of a vortex, Journal of Fluid Mechanics 525 (2005) $1-26$.

[52] L. Agostini, E. Touber, M. Leschziner, Spanwise oscillatory wall motion in channel flow: drag-reduction mechanisms inferred from dns-predicted phase-wise property variations at $r e_{\tau}=1000$, Journal of Fluid Mechanics 743 (2014) 606635.

[53] R. R. Mankbadi, Dynamics and control of coherent structure in turbulent jets, Applied Mechanics Reviews 45 (1992) 219-248.

[54] R. R. Mankbadi, Transition, turbulence, and noise: Theory and applications for scientists and engineers, volume 282, Springer Science \& Business Media, 2013.

[55] A. V. Cavalieri, P. Jordan, A. Agarwal, Y. Gervais, Jittering wave-packet models for subsonic jet noise, Journal of Sound and Vibration 330 (2011) 4474-4492.

[56] M. Kearney-Fischer, A. Sinha, M. Samimy, Intermittent nature of subsonic jet noise, AIAA Journal 51 (2013) 1142-1155.

[57] K. Viswanathan, J. Underbrink, L. Brusniak, Space-time correlation measurements in nearfields of jets, AIAA Journal 49 (2011) 1577-1599.

[58] T. Corke, S. Kusek, Resonance in axisymmetric jets with controlled helical-mode input, Journal of Fluid Mechanics 249 (1993) 307-336.

[59] J. B. Freund, Noise sources in a low-Reynolds-number turbulent jet at Mach 0.9, Journal of Fluid Mechanics 438 (2001) 277-305.

[60] R. Mankbadi, Turbulence enhancement in free shear flows under multifrequency excitation, in: Forum on Turbulent Flows-1990, volume 1, 1990, pp. 105-113. 